\title{
Coarse-grained simulations of branched bilayer membranes: effects of cholesterol-dependent phase separation on curvature-driven lipid sorting
}

\author{
Manami Nishizawa, Kazuhisa Nishizawa* \\ Department of Laboratory Medicine, Teikyo University School of Medical Technology, Tokyo, Japan; \\ *Corresponding Author: kazunet@med.teikyo-u.ac.jp.
}

Received 22 May 2011; revised 23 June; accepted 6 July 2011.

\section{ABSTRACT}

Our recent coarse-grained (CG) molecular dynamics (MD) simulations of membranes with a hemifused-ribbon ( $\lambda$-shaped) geometry showed curvature-driven demixing leading to enrichment in dioleoyl-phosphatidylethanolamine (DOPE) in a negatively-curved region (at $C \simeq$ $-0.8 \mathrm{~nm}^{-1}$ ) of a DOPE/dipalmitoyl-phosphatidylcholine (DPPC) membrane. Here we extend the analysis with respect to lipid composition and simulation time. Simulations of $12-20 \mu \mathrm{s}$ effective time show that, compared with DOPE of the DOPE/DPPC system, a DPPC/dilinoleyl$P C$ [di(18:2)PC] membrane showed a similar degree of enrichment of $\mathrm{di}(18: 2) \mathrm{PC}$ in the curved region with $C \simeq-0.8 \mathrm{~nm}^{-1}$. For the latter mixture, even weak negative curvatures $(C \simeq$ $-0.5-0.6 \mathrm{~nm}^{-1}$ ) caused significant degrees of di(18:2)PC enrichment. In agreement with recent studies of a planar bilayer, a ternary DPPC/ di(18:2)PC/cholesterol 0.42:0.28:0.3 mixture phase-separated into nanoscale raft-like liquidordered $\left(L_{o}\right)$ and non-raft liquid-disordered $\left(L_{d}\right)$ phases on a sub-microsecond time scale. The $L_{o}$ domains were preferentially localized at planar portions, whereas the $L_{d}$ domains were positioned mainly in curved regions of the membrane. Unlike binary dioleoylphosphatidylcholine (DOPC)/cholesterol and DPPC/cholesterol mixtures, which showed only a slight enrichment of cholesterol in the curved region, the ternary mixtures showed considerable migration of cholesterol and DPPC from the curved to the planar region. A pronounced degree of lipid segregation due to the preferential distribution of the $L_{d}$ and $L_{o}$ domains in the curved and planar regions, respectively, was observed even when the curvature of the fused monolayers (originally "cis" leaflets) was weakened $(C \simeq$ $\left.-0.5 \mathrm{~nm}^{-1}\right)$. Overall, the results are consistent with theoretical predictions based on spontaneous curvature of the constituent lipids and the difference in rigidity between the $L_{d}$ and $L_{o}$ domains, whereas lipid-lipid interactions, such as PE-PE or DPPC-cholesterol, as well as propensity for interleaflet colocalization (registration) of the $L_{o}$ and $L_{d}$ domains appear to significantly amplify curvature-induced lipid demixing in the $\lambda$ system. Intriguingly, for the DPPC/di(18:2)PC/cholesterol ternary mixtures, a $\mathrm{L}_{o} / \mathrm{L}_{d}$ domain boundary often moved to the branched point of the membrane, suggesting enhanced flexibility at the domain boundary. We hypothesize that curvature-driven lipid sorting and energetically favored localization of domain boundaries at sharp bends in the membranes may collaborate to assist intracellular lipid sorting.

Keywords: Lipid Raft; Stalk Formation; Membrane Rigidity; Lipid Clustering; Lipid Sorting; Lipid Mixing; Hemifusion; Cholesterol-Rich Microdomain

\section{INTRODUCTION}

Eukaryotic cell membranes contain sterols and diverse phospholipids, which differ in length and degree of acyl chain saturation [1]. Such diversity is considered to have many physiological implications for regulation of membrane structure as well as for inhomogeneous distribution of membrane constituents crucial for numerous cellular activities [2,3]. Membrane lipid diversity leads to heterogeneity in spontaneous (intrinsic) curvatures, which are likely to be important for membrane deformations such as tube formation, fission, and fusion [4]. In recent studies of cell membrane fusion, the findings of 
many experimental fusion systems [4-6] have supported the stalk-pore model. For example, early support for the stalk-pore model came from the observation that the addition of negative-curvature lipids in contacting (proximal) monolayers facilitates stalk formation, whereas the addition of positive-curvature lipids such as lysophosphatidylcholine to contacting monolayers inhibits the formation of hemifusion, a well-known fusion intermediate $[5,7,8]$. Such studies have led to a general consensus that lipids with high spontaneous curvatures may play important roles in membrane fusion.

Heterogeneity in lipid constituents permits uneven lipid distribution which may be relevant to various cell activities. Cholesterol, together with sphingomyelin (SM) or saturated lipids, forms microdomain structures such as lipid raft $[2,3,9]$. Lipid mixtures in cell membranes are considered to be in vicinity of demixing points, making raft formation dynamic and responsive to cellular states [10]. The microdomain structures not only permit the clustering of fusion proteins, such as soluble N-ethylmaleimide sensitive factor-attachment protein receptors (SNAREs), but also directly modify membrane characteristics such as fluidity, rigidity, and curvature $[9,11]$. Uneven lipids distribution should be affected by the curvature and even a weak curvature may exert a profound influence on lipid domain segregation [12-15]. Amplified segregation may, in turn, promote membrane deformation and departure of a lipid membrane from planar geometry $[12,14]$. Thus, coupling between the membrane curvature and the lipid distribution is likely to be involved in the intracellular lipid sorting that maintains the uneven lipid distribution among organelles [16-18], although the underlying mechanisms are poorly understood.

As we reviewed in our recent paper [19], a number of experimental and theoretical efforts have promoted understanding of the curvature-lipid distribution relationship [12,14,19-22]. Although curvature sorting on the basis of spontaneous lipid curvature is generally weak, curvature-dependent lipid demixing becomes pronounced when lipid mixtures are close to the demixing point (miscibility critical point) [12-14]. Because experimental and theoretical studies indicate that cholesterol and saturated lipid-rich microdomains have high rigidity, it is also interesting to see where and how such cholesterol-rich domains reside and behave in processes involving high membrane curvature, such as fusion intermediate formation $[11,23]$.

Molecular dynamics (MD) simulations have been widely used to probe the structural and dynamical properties of membranes at the atomistic level. Recent development of the coarse-grained (CG) simulation method has removed some of the difficulties associated with the time scale and system dimensions of all-atom simulations (e.g., [24,25]). The occurrence of fusion intermediates has been studied using CG simulations [26-28]. In our CG simulations, a negatively curved region of a dioleoylphosphatidylethanolamine (DOPE)/dipalmitoylphosphatidylcholine (DPPC) binary mixture membrane exhibited DOPE enrichment [19], suggesting that lipids with negative intrinsic curvature (due to an imbalance between the size of the polar headgroups and the hydrophobic tail) can be unevenly distributed, depending on the curvature of fused proximal monolayers. Such demixing of lipids may occur and stabilize the hemifusion state $[29,30]$.

Cholesterol-containing binary and ternary lipid mixtures have been analyzed using MD simulations [31-33]. Importantly, ternary lipid mixtures consisting of saturated phosphatidylcholine (PC)/unsaturated $\mathrm{PC} /$ cholesterol (to be specific, DPPC/dilinoleyl-PC [di(18:2)PC]/ cholesterol) exhibited spontaneous separation into a raftlike liquid-ordered $\left(\mathrm{L}_{\mathrm{o}}\right)$ domain and a liquid-disordered $\left(\mathrm{L}_{\mathrm{d}}\right)$ domain [34]. Our previous analysis did not consider cholesterol [19], so it is of interest to see how cholesterol-containing ternary mixtures form domains in curvature-containing lipid membranes.

Here, we extend our study to several binary and ternary lipid mixtures and discuss the effects of membrane curvature on $\mathrm{L}_{\mathrm{o}} / \mathrm{L}_{\mathrm{d}}$ phase separation. As the $\mathrm{CG}$ models used here are similar to those used in [34], we henceforth use the terms "raft-like" and "non-raft" phase (or domain) to denote the observed $\mathrm{L}_{\mathrm{o}}$ phase and $\mathrm{L}_{\mathrm{d}}$ phase (or domain), respectively (however, note that $\mathrm{L}_{\mathrm{o}}$ phases in the model membranes differ from lipid rafts in cell membranes in many points, as discussed by, e.g., [35]). To address these issues, $\lambda$-shaped membranes are used (Figure 1); they consist of two bilayer ribbons that are partly fused, producing a $\lambda$-shaped cross section. Results show that DPPC/di(18:2)PC/cholesterol mixtures exhibit nanoscale phase separation and that the raft-like $\mathrm{L}_{\mathrm{o}}$ domains are preferentially localized in the planar regions of the $\lambda$ system, whereas $L_{d}$ domains are positioned mainly in the curved region. In this study, we present the main results in the Results section. Several other notable findings are presented and discussed in the Discussion section. We intend to address several relevant issues, such as lipid diffusion dynamics and the tension imposed on the tethering molecules (i.e., the force required for membrane bending), in our forthcoming paper.

\section{SIMULATION DETAILS}

\subsection{Simulation Parameters}

The GROMACS 3.3.1 suite was used for all simulations [36]. The CG representation was performed using 


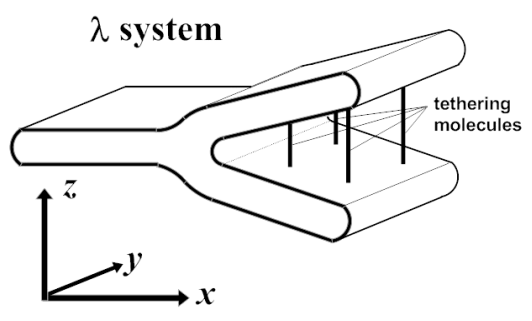

(a)

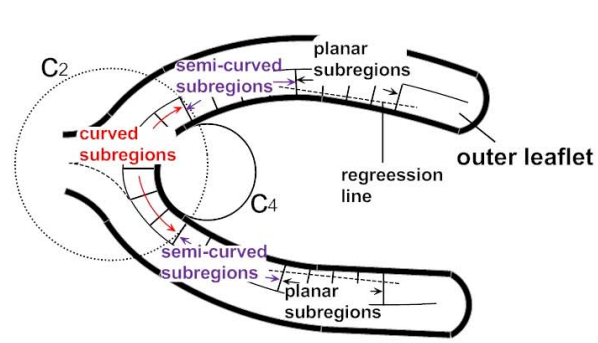

(c)

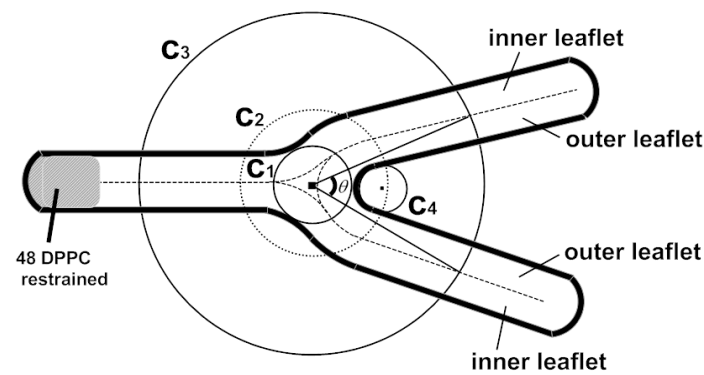

(b)

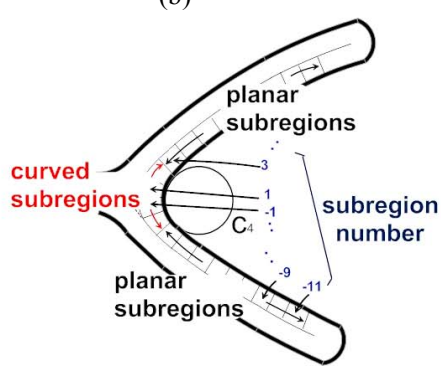

(d)

Figure 1. Branched bilayer membrane system ( $\lambda$ system). (a) Schematic view. The $\lambda$-shaped (hemifused) bilayer ribbons have infinite length in the $y$-direction because of the periodic boundary condition. For clarity, water is hidden. Tethering molecules are shown, but the positions are incorrect; (b) Definition of curvature strength and angle between two fused leaflets based on the circles C1-C4 derived as follows. $\mathrm{C} 1$ has the longest radius but is located inside of any phosphorus atoms of any lipid monolayer. $\mathrm{C} 2$ and $\mathrm{C} 3$, the common center of which is the same as that of $\mathrm{C} 1$, are then drawn; in this study, their radii were fixed at $4 \mathrm{~nm}$ and $12 \mathrm{~nm}$, respectively. Bilayer midplanes (broken lines) of each of the three bilayers are based on the lipid atom coordinates projected onto the $x$-, $z$-plane. Points of intersection between $\mathrm{C} 3$ and the midplanes define the angle $\theta$. C4 is the circle that best fits the phosphorus atoms that belong to the outer leaflet and are located inside of $\mathrm{C} 2$. Radius of $\mathrm{C} 4$ represents the negative curvature of the outer leaflet; (c) Schematic representation of curved, semi-curved and planar subregions. For definition, see Methods; (d) Illustrated example of the $\lambda$ membrane with a small curvature. The scheme for subregion numbering is also illustrated in blue.

the MARTINI forcefield (ver 2.0) by Marrink and coworkers [37]. For example, for DPPC, the choline was represented by Q0 particles and the phosphate group was represented by Qa, whereas the glycerol ester was represented by two Na particles. Each of the lipid tails was modeled by four $\mathrm{C} 1$ particles. For DOPE, four $\mathrm{C} 1$ particles and one $\mathrm{C} 3$ particle represented the acyl chain, whereas Qa and Qd particles represented PO4 and ethanolamine, respectively. For di(18:2)PC, each acyl chain was represented by a $\mathrm{C} 1-\mathrm{C} 4-\mathrm{C} 4-\mathrm{C} 1$ chain. Four water molecules were modeled by one particle, as in [37]. In the CG simulations, the Lennard-Jones interactions were smoothly shifted to zero between $0.9 \mathrm{~nm}$ and $1.2 \mathrm{~nm}$ to reduce the cut-off noise, and the electrostatics were smoothly shifted to zero between 0 and $1.2 \mathrm{~nm}$; a relative dielectric constant of 15 was used for explicit screening of the Coulombic energy function. The time step for integration was $20 \mathrm{fs}$, and the non-bonded neighbor list was updated every 10 steps. Systems were maintained at a temperature of $323 \mathrm{~K}$ for set $1-3$ and 295 $\mathrm{K}$ for set 4-13 (Table 1). For the $295 \mathrm{~K}$ simulations anti- freezing molecules (WF particles [37]) were added to water at $10 \mathrm{~mol} \%$. For the temperature control, three groups (water, phospholipids, and cholesterol) at maximum were separately coupled with a constant $\tau_{\mathrm{T}}$ of 0.1 ps and relaxation time of $0.1 \mathrm{ps}$ using Berendsen coupling [38]. We chose the group coupling scheme because of frequent freezing of water in the $\lambda$ system that we observed when coupling the membrane as a group. Of note, our pilot planar membrane simulations showed no difference in the time evolution and the properties of the domains between the non-grouping method and the grouping method that divided the membrane into four groups (details not shown). So, although artifacts caused by such group coupling cannot be formally ruled out, we believe that such effects are negligibly small for our present study. The system pressure was anisotropically coupled using the Berendsen algorithm at 1 bar with a coupling constant $\tau_{\mathrm{P}}=0.2 \mathrm{ps}$ and a compressibility of 3 $\times 10^{-5} \mathrm{bar}^{-1}$. The simulation time in this study is indicated after multiplication by a factor of 4 , as in [37], to account for the large diffusion rates because of the 
Table 1. Simulations in the $\lambda$ system. Set 1-3 were run at $323 \mathrm{~K}$. The others were run at $295 \mathrm{~K}$. ${ }^{a}$ Overall compositions are shown. During simulations, lipid composition of the outer leaflet deviated slightly from the value due to interleaflet translocations of lipids. ${ }^{\mathrm{b}}$ The result of a representative trajectory is shown. C4_radius and theta are shown with s.d. obtained from final $1 \mu$ s of the trajectory. ${ }^{c}$ Lipid mol\% in curved and planar subregions are shown. "Curved" here shows the sum over the curved and semi-curved subregions.

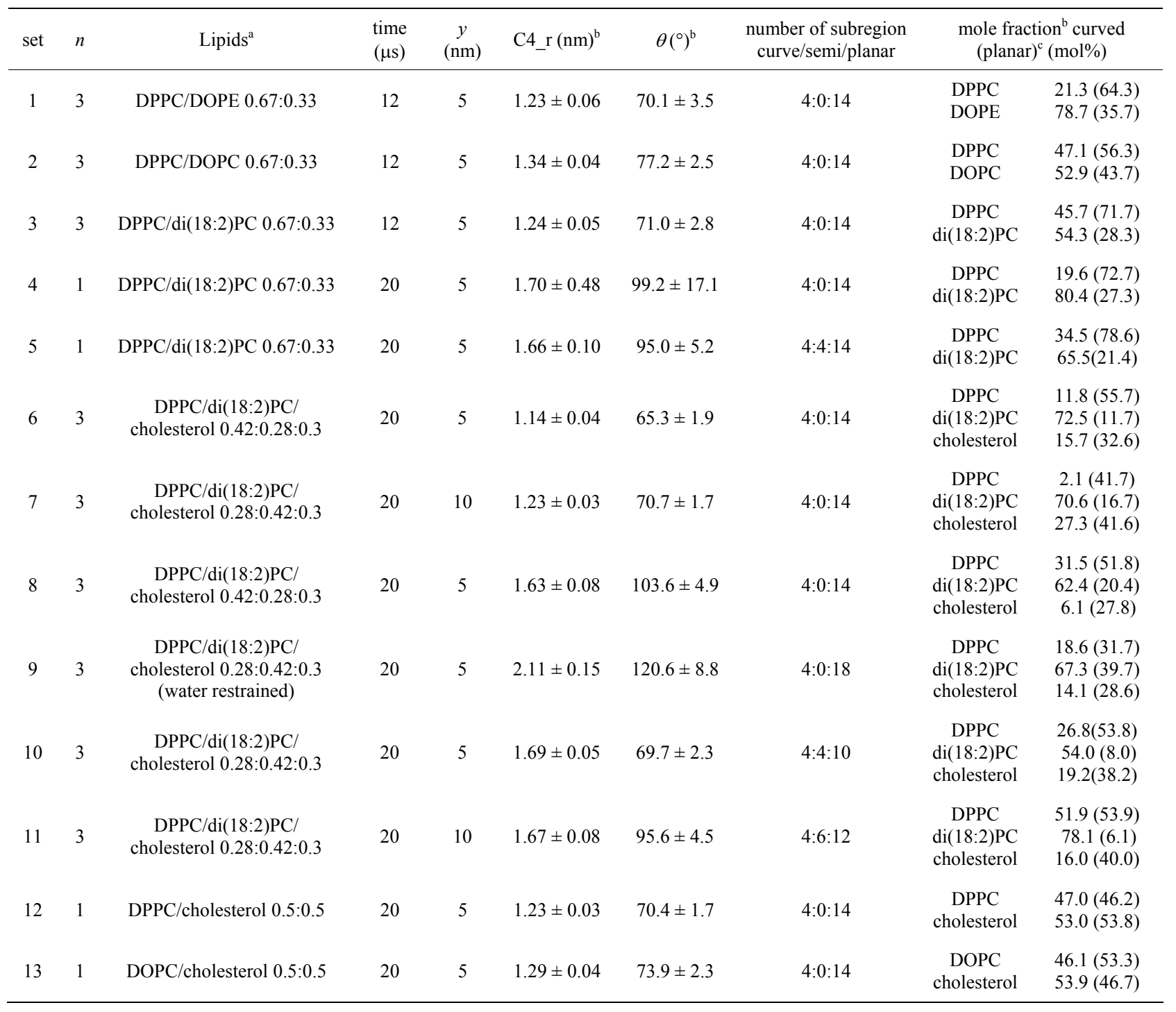

smoothed surface of molecules in the CG system.

\subsection{Branched Membrane System}

The $\lambda$ system described above was used as the membrane geometry [19] (Figure 1(a)). The directions of the $x$-, $y$-, and $z$-axes are shown in Figure 1(a). The outer leaflet is defined as the hemifused proximal (cis) monolayers (Figure 1(b)). The $\lambda$ membrane is surrounded by water and has infinite length in the $y$ direction because of the periodic boundary condition. The planar regions and curved region of the outer leaflet and the subregions of the planar and curved regions are shown in Figure 1(c) and $d$ and in the following section.
The strength of the (negative) curvature of the outer leaflet is represented by the angle $\theta$ and the radius of $\mathrm{C} 4$. $\theta$ represents the angle between the two bilayers (Figure 1); a small $\theta$ value corresponds to a sharply negative bending of the outer leaflet. To maintain the curvature at a desired strength, artificial tethering molecules linked to two DPPC molecules were placed between the cis monolayers (Figure 1(a)). The tethering molecules were named LK peptides and consist of appropriate numbers of Ser residues. Each Ser residue was represented by P1 (peptide backbone) and P5 (sidechain) particles [19]. The central particles of the LK peptides were harmonically restrained at appropriate positions. For example, for set 1, four LK3 peptides (i.e., LK peptides composed 
of three Ser residues) were restrained at a position $4 \mathrm{~nm}$ along the $x$ direction from the center of circle $\mathrm{C} 1$, whereas two LK6 peptides were restrained at $8 \mathrm{~nm}$ and two more at $6 \mathrm{~nm}$ from the center of $\mathrm{C} 1$. We chose to use position restraints on the $\mathrm{Na}$ particles (that juxtaposed the Qa particles) of several ( 48) lipids at the leftmost edge, as our attempt at using internal coordinates based on the primary axis of inertia still resulted in large fluctuations in the membrane structure. However, such restraints should result in various artifacts, requireing further analysis to address these artifacts.

Simulation sets consisted of various numbers of lipids and water molecules (Table 1). The number of water molecules ranged from $\sim 11,400$ (set 1-3) to $\sim 30,000$ (set 11). Accordingly, the approximate simulation box size also varied, ranging from $(x, y, z)=(31,5,16)$ to $(x, y, z)$ $=(30,10,24)(\mathrm{nm})$. Calculations were carried out on 35 Intel Core 17 processors. All molecular images were constructed using Visual Molecular Dynamics [39]. designations.

\subsection{Analysis}

The local properties (composition, thickness and the order parameter) of planar bilayers were computed by dividing the membrane into slabs of $1 \mathrm{~nm}$ width in the direction perpendicular to the domain boundaries or into $1 \mathrm{x} 1 \mathrm{~nm}$ square segments. Both segmentation methods yielded the essentially similar results, but we chose the square segmentation scheme for the planar (Figure 2) and the slab segmentation scheme for the curved membranes (Table 1, set 1-12). For the local membrane thickness, the distance along the membrane normal between the average position of the particle of the terminals of lipid tails (C4Bs for DPPC and di(18:2)PC or C5Bs for DOPE and DOPC) and that of the PO4s (representing the phosphate group) within each slab was taken as the thickness. The lipid order parameter was calculated using the vector averaged over all four (for DPPC and di(18:2)PC) or five (for DOPE and DOPC) tail segments of all lipid tails present in that slab [34].

For the local property analysis of the outer leaflet of the $\lambda$ membranes, the leaflet was divided into $1 \mathrm{~nm}$-wide subregions with boundaries parallel to the $y$-axis (Figure 1(c)). Using the following scheme, the subregions were classified into "curved", "semi-curved" and "planar" subregions. First, the outer leaflet portion located inside $\mathrm{C} 2$ was divided such that each subregion stands on a 1 $\mathrm{nm}$ portion of the $\mathrm{C} 4$ circumference; by excluding the residual, as many subregions as possible (typically four) were obtained (Figures 1(c) and (d)). Subsequently, by finding the regression line to the phosphorus particles (projected onto the $x, z$-plane) of the lipids outside the circumference of $\mathrm{C} 2$, a regression plane, which was par- allel to the $y$-axis and represented the planar portions, was found. Those outer leaflet portions located outside of and $0-9 \mathrm{~nm}(0-11 \mathrm{~nm}$ only for set 5, 9 and 11) away from the $\mathrm{C} 2$ circumference were divided into several subregions (each with a length of $1 \mathrm{~nm}$ along the plane) and were tentatively considered planar subregions; however, when the regression plane fit poorly (as in the case of a horseshoe-like curvature), the subregions adjacent to the circumference of C2 were redefined as semicurved subregions and excluded, and the plane-fitting against fewer distal subregions was performed. This procedure (i.e., redefinition and exclusion of semi-curved subregions and plane refitting to the remaining subregions) was iterated until the fitting met the criterion that, for all planar subregions, the mean plane phosphorus atom distance was $<0.5 \mathrm{~nm}$. Those outer leaflet portions distant from the circumference of $\mathrm{C} 2(>9 \mathrm{~nm}$, but $>11$ $\mathrm{nm}$ for set 5, 9 and 11) were excluded from the local property analysis. Depending on the overall geometry of the membrane, the numbers of curved, semi-curved and planar subregions differed among the simulations; typically, the outer leaflet was divided into 4 curved subregions, 0 - 6 semi-curved subregions and $10-18$ planar subregions. The local properties (composition, thickness and the order parameter) of the outer leaflet were calculated for each subregion, where the membrane normal served as the reference direction for thickness and the order parameter. For the curved subregions the normal was calculated using the $\mathrm{C} 4$ circumference, whereas, for semi-curved subregions and planar subregions, the normal was determined based on the regression plane calculated for each subregion using the xz-projected positions of the phosphorus atoms. Thus obtained membrane normal was used for the thickness measurement, based on the same procedure as for the planar membranes.

\section{RESULTS}

In this study, the local properties (composition, thickness, and order parameters) of lipid monolayers in the $\lambda$ membranes are analyzed. As a reference, these properties were first evaluated for the monolayers contained in a standard planar bilayer membrane. Our simulation of a DPPC/di(18:2)PC/cholesterol 0.42:0.28:0.3 planar bilayer of $10 \times 20 \mathrm{~nm}$ size reproduced the coexisting liquid domains $\left(\mathrm{L}_{\mathrm{o}}\right.$ and $\left.\mathrm{L}_{\mathrm{d}}\right)$ as reported in [34]. The $\mathrm{L}_{\mathrm{o}}$ domains had typical compositions of DPPC/di(18:2)PC/ cholesterol 0.55: 0:0.45 (thus, devoid of $\operatorname{di}(18: 2) \mathrm{PC}$ ), compared to the $\mathrm{L}_{\mathrm{d}}$ domains with $\sim 0: 0.85: 0.15$ (devoid of DPPC) (Figure 2(a)). When representative $1 \times 1 \mathrm{~nm}$ squares located in the $\mathrm{L}_{\mathrm{d}}$ (and $\mathrm{L}_{\mathrm{o}}$ ) domains were sampled, the bilayer membrane thickness (measured as the mean $z$-coordinate of all PO4 particles located in a slab of 1 $\mathrm{nm}$ width) was $3.62( \pm 0.01) \mathrm{nm}$ for the non-raft $\mathrm{L}_{\mathrm{d}}$ phase 
and $4.48( \pm 0.02) \mathrm{nm}$ for the raft-like $\mathrm{L}_{\mathrm{o}}$ phase at $20 \mu \mathrm{s}$ in agreement with [34]. When a compositionally asymmetrical bilayer [specifically, a bilayer consisting of a DPPC/di(18:2)PC/cholesterol (i.e., ternary) monolayer and a dioleoylphosphatidylcholine (DOPC)/cholesterol (binary) monolayer] was simulated, the raft-like $\mathrm{L}_{\mathrm{o}}$ domains formed only in the ternary monolayer (Figure 2(b)). Figures 2(c) and (d) shows the thickness and order

(a)

$$
\text { symmetrical bilayer }
$$
of DPPC/di(18:2)PC/cholesterol

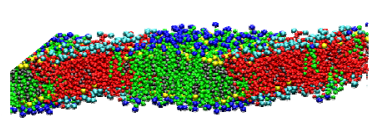

(c)

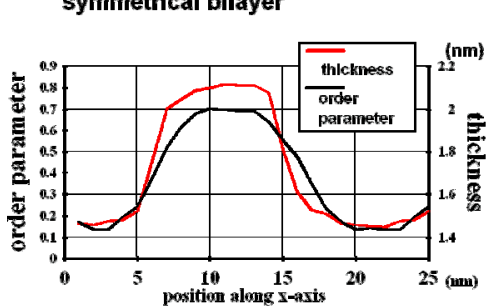

(e)

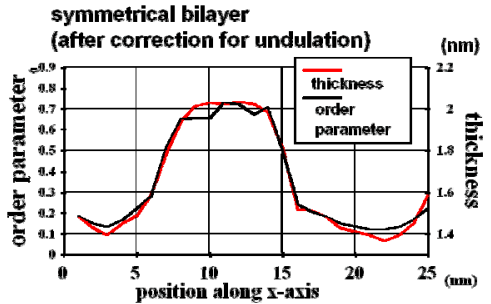

(g)

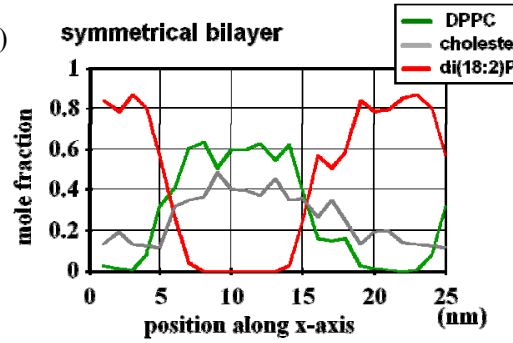

parameter of the upper monolayer of the bilayers shown in Figures 2(a) and (b), respectively. (Figures 2(e) and (f) are similar to Figures 2(c) and (d), but the influence of the membrane undulation was reduced by using the membrane normal of each $1 \mathrm{~nm}$ slab for calculation.) The thickness and order parameter of the $\mathrm{L}_{\mathrm{o}}$ phase formed in the upper monolayer of the asymmetrical membrane (Figures 2(d) and (f)) were close to the values from the

b) asymmetrical bilayer

upper monolayer : DPPC/di(18:2)PC/cholesterol

lower monolayer : DOPC/cholesterol

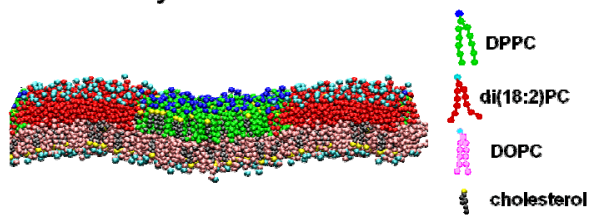

(d)

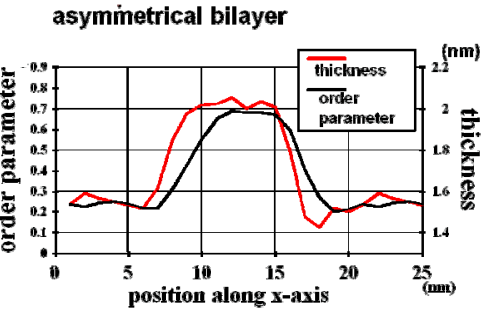

asymmetrical bilaye

(f)
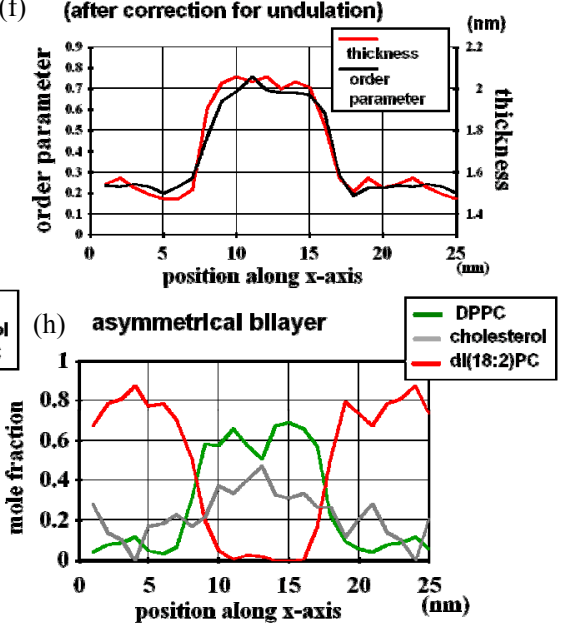

Figure 2. Planar bilayer simulations and the local properties of $\mathrm{L}_{\mathrm{o}}$ and $\mathrm{L}_{\mathrm{d}}$ domains. (a) Side view of the DPPC/di(18:2)PC/cholesterol simulation at the end (20 $\mu \mathrm{s})$ of the simulation. Representation scheme; blue spheres, DPPC headgroup particle NC3; green spheres, DPPC particles other than NC3; cyan spheres, di(18:2)PC headgroup particle NC3; red spheres, di(18:2)PC particles other than NC3; yellow sphere, cholesterol OH group particle; gray spheres, cholesterol particles other than $\mathrm{OH}$ group. Water particles are not shown for clarity. (b) Side view of a planar bilayer consisting of a DPPC/di(18:2)PC/cholesterol 1:1:1 monolayer and DOPC/cholesterol 1:1 monolayer at the end of the simulation. Representation scheme is same as (a), but DOPC tails and headgroups are shown with pink and cyan spheres, respectively. (c) Local properties of the upper monolayer of the membrane shown in (a). Thickness and order parameter of $1 \times 1 \mathrm{~nm}$ square patches (along the $x$-axis) of the upper monolayer are shown. Red line, thickness; black line, order parameter. (d) Same as in (c) but the upper monolayer of (b) is analyzed. (e) and (f) Same as (c) and (d), respectively, but the properties calculated against the membrane normal determined for each subregion are shown. (g) and (h) Mole fraction analysis of the upper monolayer of the membranes shown in (a) and (b), respectively. 
symmetrical raft-like domains (Figures 2(c) and (e)). Although rigorous statistical analyses to address the question whether the boundary of the $\mathrm{L}_{\mathrm{o}}$ domains forming in the asymmetric bilayer was less sharp than those in the symmetric bilayer are beyond the scope of this study, our compositional analysis showed that both of the membranes formed similarly sharp $\mathrm{L}_{\mathrm{o}}$ boundaries (Figures 2(g) and (h)).

For comparison, the thickness of the pure DPPC bilayer was $4.19( \pm 0.02) \mathrm{nm}$ (PO4-PO4 distance), whereas that of each monolayer is $1.89( \pm 0.01) \mathrm{nm}(\mathrm{PO} 4-\mathrm{C} 4 \mathrm{~B}$ distance). These values are smaller than those for the $\mathrm{L}_{0}$ domains $(\sim 2.0 \mathrm{~nm})$ analyzed in Figure 2, demonstrating that the well-studied effects (i.e., membrane-thickening and lipid-ordering effect) induced by interactions between cholesterol and saturated lipid. For the DOPC bilayer, these values were $4.47( \pm 0.03) \mathrm{nm}(\mathrm{PO} 4-\mathrm{PO} 4)$ and $1.98( \pm 0.01) \mathrm{nm}(\mathrm{PO} 4-\mathrm{C} 5 \mathrm{~B})$, respectively. For the DOPE bilayer, these values were $4.62( \pm 0.01)$ and 2.04 $( \pm 0.01) \mathrm{nm}$, respectively. However, the large values of these bilayers can be ascribed to the acyl chains, which are represented by five particles, unlike the di(18:2)PC and DPPC, which are modeled by only four particles. For di(18:2)PC bilayer the corresponding values were $3.46( \pm 0.02)$ and $1.42( \pm 0.01) \mathrm{nm}$, respectively.

For the convenience in thickness measurement in curved membranes, we tentatively define the $\mathrm{L}_{0}$ monolayer domain as the $1 \mathrm{~nm}$ slab with a high DPPC and cholesterol mol\% (both $>0.35$ ), with thickness $>2.0 \mathrm{~nm}$ and with the order parameter $>0.65$. We also tentatively define the $\mathrm{L}_{\mathrm{d}}$ monolayer domain of the ternary mixture as the region with thickness $<1.6 \mathrm{~nm}$ and the order parameter $<0.3$. On the other hand, for lipid mixtures containing DOPC (or DOPE), the long acyl chains of the used CG models make the thickness-based phase definition inappropriate. Also, for the binary phospholipid mixtures (DPPC/ DOPE, DPPC/DOPC and DPPC/di(18:2)PC) our preliminary thickness and order parameter analysis was not particularly informative beyond composition analysis (details not shown).

So, in this study we did not examine the thickness and the order parameter for these binary membranes.

\subsection{Curvature-Sorting in Binary Lipid Mixtures}

Table 1 summarizes the geometry and lipid distribution at the end of the main simulations. Binary phospholipid mixtures without cholesterol were examined first (sets 1-5).

When our recent simulation of a DPPC/DOPE 0.67: 0.33 mixture [19] at $323 \mathrm{~K}$ was extended to $12 \mu \mathrm{s}$, further enrichment in DOPE in the region was observed
(78.7 $\mathrm{mol} \%$ in curved subregions and $35.7 \mathrm{~mol} \%$ in planar subregions, more uneven compared with $62.6 \mathrm{~mol} \%$ and $37.8 \mathrm{~mol} \%$, the corresponding values at $2 \mu \mathrm{s}$ [19]) (Table 1, set 1). (We note that interleaflet migrations of lipids allow the outer leaflet lipid composition to deviate from the overall lipid composition.) Figure 3(a) (left) shows the number of each lipid species contained in each subregion (defined as in Methods and Figure 1)). The DOPE enrichment was accounted for by a limited number ( 2 or 3 ) of subregions that curved strongly. In a similar setting, the curved subregions of the outer leaflet of the DPPC/DOPC 0.67:0.33 membrane exhibited only slight enrichment in DOPC $(52.9 \%$ for curved and 43.7\% for planar subregions) (Table 1, set 2 and Figure 3(b)), although the possibility that the system was not equilibrated cannot be ruled out. For the DPPC/di(18:2)PC 0.67:0.33 mixture (set 3), a weak separation was observed (Figure 3(c)). However, at $12 \mu$ s the di(18:2)PCrich subregions were not exactly overlapped to the curved subregions (Figure 3(c)). Despite this phenomenon, di(18:2)PC reached $54.3 \%$ in curved subregions, but $28.3 \%$ in the planar subregions. (Also note that in set 4 and 5 di(18:2)PC also showed a relatively high degree of demixing, as shown below.) These results show the importance of the difference in spontaneous curvature among constituents as well as lipid-lipid interactions in curvature-driven lipid sorting in binary mixtures. Notably, the DPPC/di(18:2)PC mixture result (set 3) shows that heterogeneity in headgroup-headgroup interaction is not a prerequisite; rather, differences in acyl chain structure alone can lead to extensive curvature sorting.

In the analyses described above, a high curvature was maintained by the tethering molecules. It is relevant to ask whether lesser (weak) curvatures can promote lipid sorting. We note that a weak curvature can represent either of two types of curvature: a curvature with a large $\theta$ value but a modest $\mathrm{C} 4$ radius (Figure 1(d)) or alternatively, a horseshoe-like curvature with a large $\mathrm{C} 4$ radius and a modest $\theta$ value (Figure 1(c)). Although we found it difficult to control these features independently, care was taken to cover both types. For easy comparison with [34], the simulations described below were run at $295 \mathrm{~K}$.

When the tethering molecules were removed from the DPPC/di(18:2)PC simulation, the curvature quickly weakened ( $\sim 400 \mathrm{~ns}) ; \theta$ and the $\mathrm{C} 4$ radius reached $\sim 99^{\circ}$ and $1.7 \mathrm{~nm}$, respectively (set 4). A pronounced degree of di(18:2)PC enrichment in curved subregions occurred $80.4 \%$ compared with $27.3 \%$ in the planar subregions (Figure 4(a)).

When the same mixture was subjected to a horseshoe-like membrane in which the crosslinking molecules were placed at distal positions to hold the structure, the outer monolayer still exhibited a significant degree of 
demixing of the DPPC/di(18:2)PC mixtures at $20 \mu \mathrm{s}$ (Figure 4(b), set 5) and the di(18:2)PC mole fraction in the curved/semi-curved subregions was about 3-fold of that in the planar subregions, yet this was less pronounced compared with some of the cholesterol-containing ternary mixtures (examined below). We have not examined very high curvature lipids such as diacylglycereol (DAG), whose sorting could be relevant in various physiological situations.

\subsection{Cholesterol-Containing Ternary Mixtures}

As shown above, our analysis reproduced the finding of [34] that the DPPC/di(18:2)PC/cholesterol mixture phase separates into $L_{o}$ and $L_{d}$ phases in planar mem- branes. To examine how the phases are associated with the membrane curvature, a DPPC/di(18:2)PC/cholesterol 0.42:0.28:0.3 mixture was simulated in a $\lambda$ system (set 6), starting from a random lipid allocation. The $L_{o}$ and $\mathrm{L}_{\mathrm{d}}$ domains formed on a timescale similar to that in the planar simulation.

By $\sim 10 \mu$ s most $\mathrm{L}_{d}$ domains were found in the curved regions, whereas most $\mathrm{L}_{\mathrm{o}}$ domains appeared in the planar regions (Figure 5(a)). Correspondence among the local properties (thickness/order parameter and mole fraction) was the essentially same as that in the planar simulations (details not shown). At $20 \mu$ s, the composition of DPPC/ $\operatorname{di}(18: 2) P C /$ cholesterol was 0.12:0.73:0.16 for the curved subregions and 0.56:0.12:0.33 for the planar subregions, showing 6-fold enrichment of $\operatorname{di}(18: 2) \mathrm{PC}$ in the curved
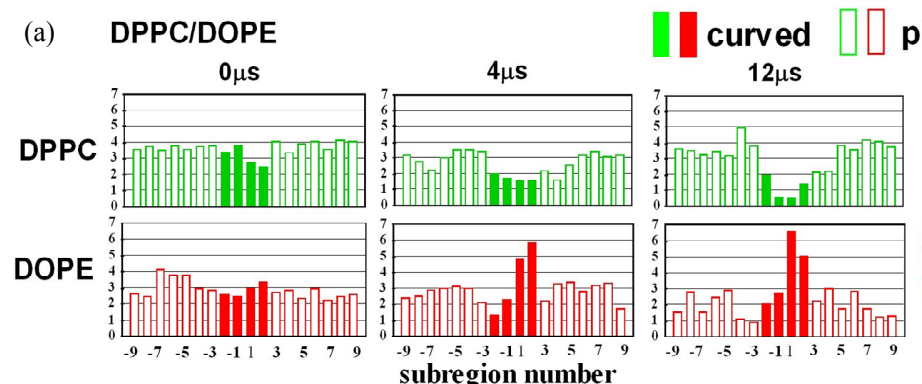

\section{planar}
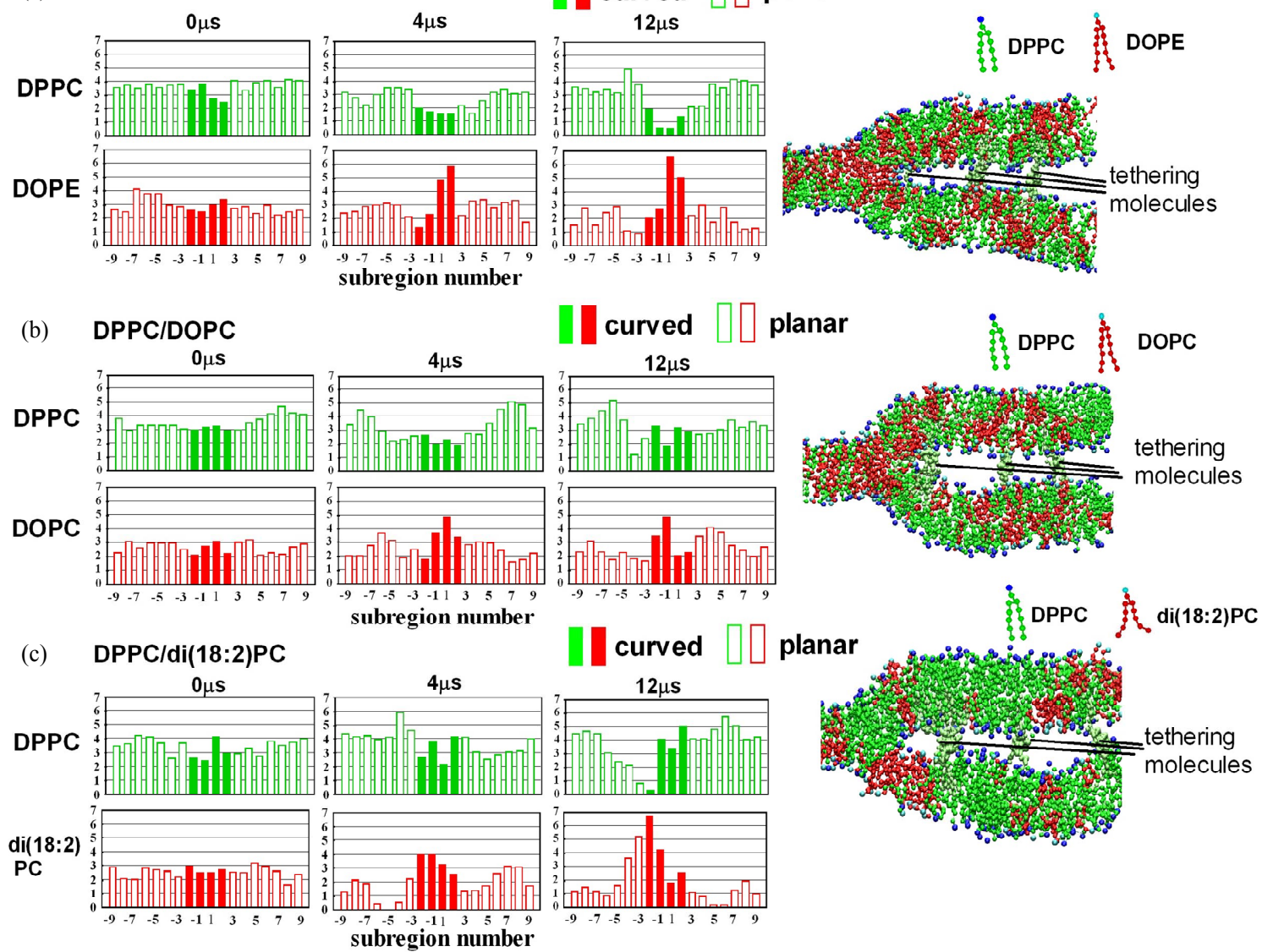

Figure 3. (a)-(c) Lipid distribution and local properties of the binary lipid mixtures simulated in the $\lambda$ membrane. For each set, the data of a representative trajectory are shown. Left, lipid composition of each subregion of the outer leaflet. Each bar shows the average over the $200 \mathrm{~ns}$ immediately before the indicated time point. Classification of subregions (i.e., curved and planar) is also shown by different styles in the histogram bars. Right, side view at $20 \mu \mathrm{s}$ (set 1 of Table1) of the molecules contained in a $1 \mathrm{~nm}$-thick slice whose plane is vertical to the $y$-axis. (a) DPPC/DOPE mixture (set 1 in Table 1); (b) DPPC/DOPC mixture (set 2); (c) DPPC/di(18:2)PC mixture (set 3). Presentation scheme for side views in (a)-(c): blue spheres, DPPC headgroup particle NC3; green spheres, non-NC3 DPPC particles; cyan spheres, headgroup particle [NH3 of DOPE and NC3 of di(18:2)PC and DOPC]; red spheres, non-NH3 DOPE particles and non-NC3 particles of di(18:2)PC and DOPC; lime surfaces, tethering molecules and the DPPC molecules to which the tethering molecules anchor directly. Water particles are hidden for clarity. 
(a)

DPPC/di(18:2)PC, wide $\theta$

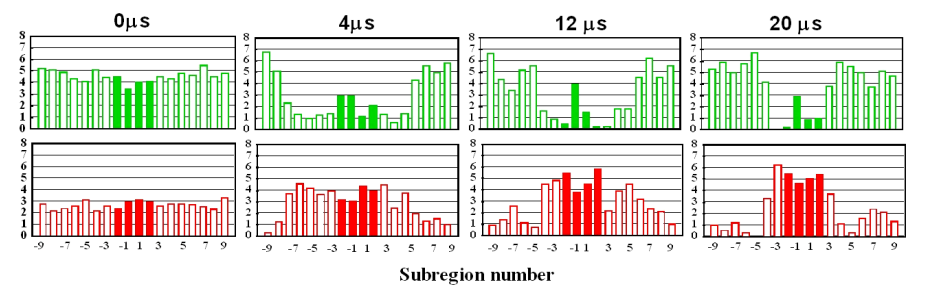

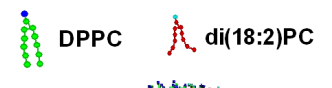
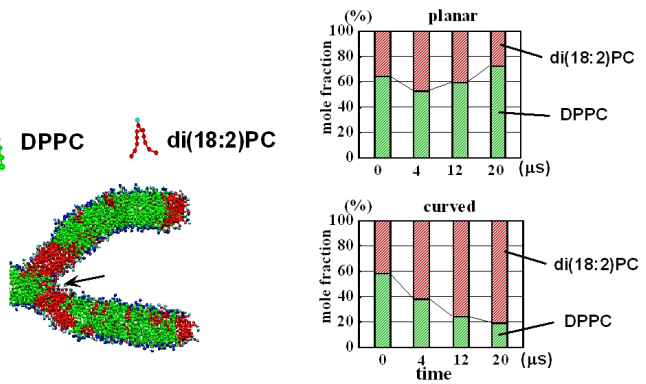

(b) DPPC/di(18:2)PC, horseshoe-like

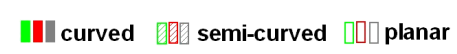
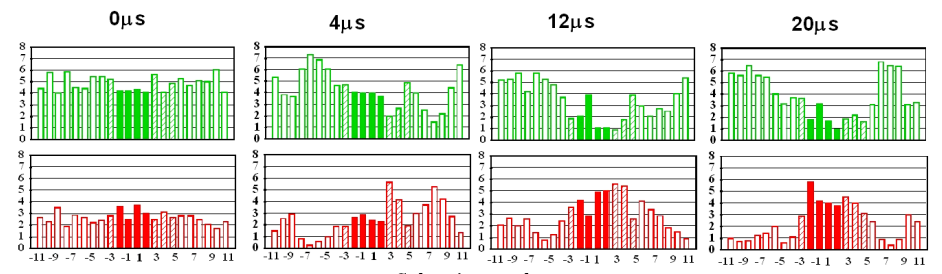

Subregion number

(c) mole fraction profile
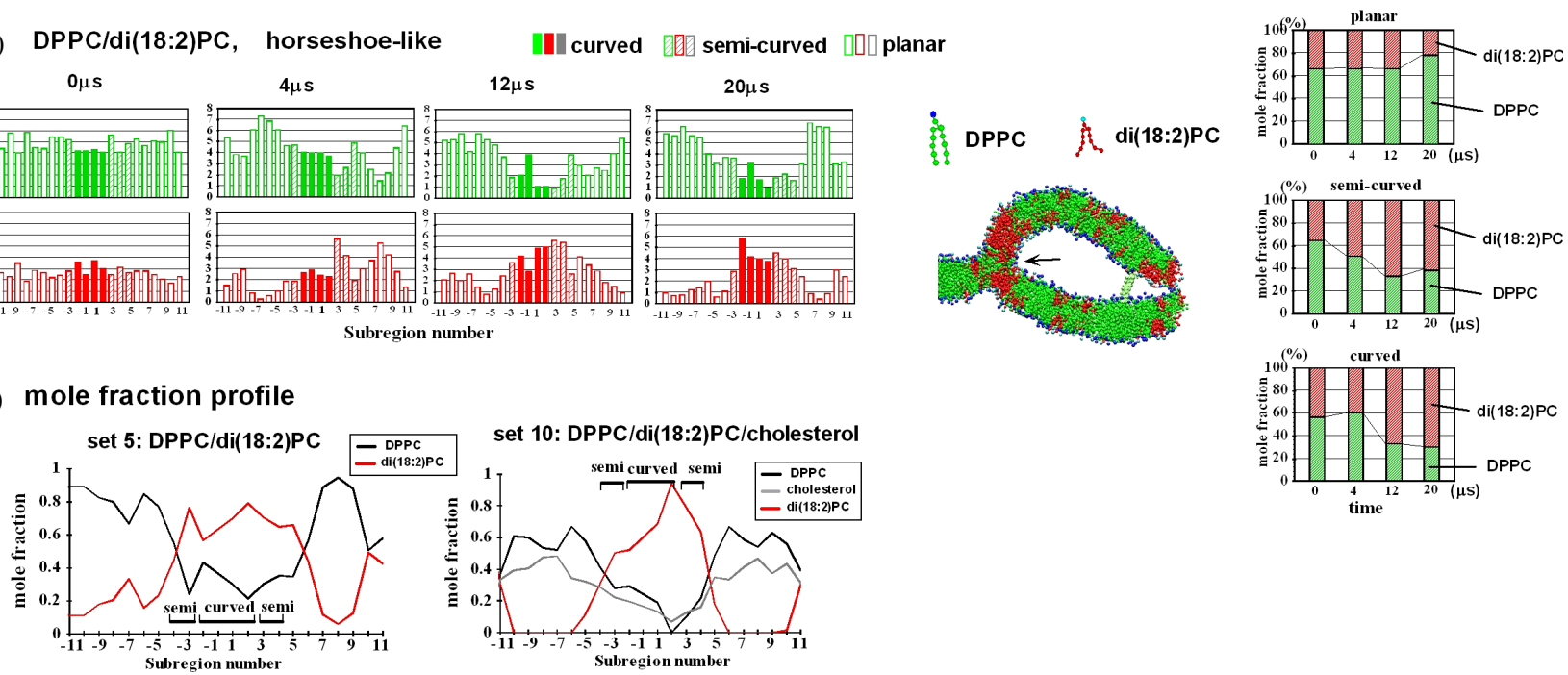

Figure 4. Lipid distribution of the DPPC/di(18:2)PC mixture of a $\lambda$ system with weak curvature. Presentation scheme is the same as in Figure 3(c), but in the right, time-dependent change of the components of the subregions are shown. (a) Distribution in the outer leaflet with a wide $\theta$ (set 4); (b) Distribution in the outer leaflet with a horseshoe-like curvature (set 5). Arrows in (a) and (b) highlight DPPC molecules located at the branching point of the membrane; (c) Lipid mole fraction of each subregion. Representative results are shown. Bars labeled "curved" and "semi" indicate the curved and semi-curved subregions respectively. Left, a DPPC/di(18:2)PC simulation of set 5. Right, a DPPC/di(18:2)PC/cholesterol ternary mixture simulation of set 10. Note that in the ternary but not in the binary simulation, lipid demixing leads to local depletion of DPPC/cholesterol or di(18:2)PC in several subregions.

compared with the planar subregions (Table 1, set 6). Thus, the DPPC/di(18:2)PC/cholesterol mixture, which has a strong demixing propensity [34], exhibits curvature-associated phase positioning at a curvature of $C=$ $\sim-0.9$. When the $y$-dimension of the membrane was doubled to reduce the finite size effect and the overall composition was altered to $0.28: 0.42: 0.3$, the result was similar (set 7).

The same mixture (i.e., DPPC/di(18:2)PC/cholesterol) was also simulated in a weakly curved $\lambda$ system. When the tethering molecules were removed (set 8 , Figure 5(b)), uneven positioning of the domains $\left(\mathrm{L}_{\mathrm{o}}\right.$ in planar subregions and $\mathrm{L}_{\mathrm{d}}$ in curved subregions) was observed; in a representative run, $\operatorname{di}(18: 2) \mathrm{PC}$ was at $62.4 \%$ in the curved and semi-curved subregions and $20.4 \%$ in the planar subregions at $20 \mu$ s.

To avoid the fluctuation effect, positional restraints of $10 \%$ of water molecules in the simulation box were further introduced (set 9). This maintained $\theta$ at $\sim 120^{\circ}$. Still, the pronounced demixing was observed (Table 1). Strikingly, when the mixture was simulated in a horseshoe-like curvature system (set 10, Figure 5(c)), the outer leaflet exhibited pronounced unevenness in the $\mathrm{L}_{\mathrm{d}}$ and $\mathrm{L}_{\mathrm{o}}$ domain distribution and hence in the DPPC and di(18:2)PC distribution, similar to set 6 . The result was similar when the $y$-dimension of the membrane was doubled (set 11). The result was not likely to have arisen from artifacts due to the presence of edges (rightmost part of the membrane) because several domain boundaries formed in the subregions distant from the edges (e.g., Figure 7). This suggests that even a weak (lesser) curvature $\left(C=-0.6 \mathrm{~nm}^{-1}\right)$ can cause a dramatic curvatureassociated distribution of the $\mathrm{L}_{\mathrm{o}}$ and $\mathrm{L}_{\mathrm{d}}$ domains in a cholesterol-containing ternary mixture, although the intermonolayer registration of the $\mathrm{L}_{\mathrm{o}}$ domains (and possibly $\mathrm{L}_{\mathrm{d}}$ domains as well) could be an important factor assisting the biased domain distribution of set 10 and 11 , as considered in Discussion. In any case, compared with 
the di(18:2)PC/DPPC binary mixture results (sets 3,4 and 5), the ternary results (sets 6 and 8-11) show that the cholesterol-dependent domain formation generally facilitates lipid demixing. It was also noted that, because of the sharp $\mathrm{L}_{0} / \mathrm{L}_{\mathrm{d}}$ domain boundaries, the composition of subregions changed abruptly within a few adjacent subregions in the case of the ternary mixtures, whereas the di(18:2)PC/DPPC binary mixture showed a relatively smooth change in composition. For example, the di(18:2)PC-rich region of the ternary simulation (set 10) formed a clear boundary and also almost led to the depletion of DPPC and cholesterol (e.g., Figure 4(c), right), whereas di(18:2)PC-rich regions in the binary mixture simulation (set 5) formed less clear boundaries (Figure 4(c), left) and contained a substantial number of DPPC molecules, despite the similar membrane geometry (horseshoe-like) of these sets. This demonstrates the effect of cholesterol that assists demixing of lipids.

Intriguingly, in one simulation of set 8 and one of set 10, despite the di(18:2)PC enrichment in the curved subregions at $4 \mu \mathrm{s}$, some domain boundaries formed at the branching point of the $\lambda$ membrane between 4 and 20 $\mu$ s (Figure 7(a) and (b)). It is possible that $\mathrm{L}_{\mathrm{o}} / \mathrm{L}_{\mathrm{d}}$ domain boundaries have anomalously small bending stiffness and the location at the branching point could be energetically favored. Another notable finding was that frequent presence of a small number of DPPC molecules, along with two cholesterol molecules, at the branching point, which could be explained by an energetically favored distribution of longer tailed lipids near the void (e.g., arrows in Figures 4(a), (b), 5(b) and 7(a)).

For set 6-11, the sum of the DPPC and cholesterol mol\% exhibited a strong correlation with the thickness and the order parameter for each subregion; $r^{2}=0.78$ (s.d. $=0.04)$ for the thickness and 0.77 (s.d. $=0.05$ ) for the order parameter, which were slightly smaller than 0.79 and 0.83 , the values for the planar monolayers shown in Figure 2, respectively. However, about a half of the subregions with high DPPC/cholesterol density (with the sum $>0.95$ ) were thinner and had a smaller order parameter than our criteria of the $\mathrm{L}_{o}$ phase, suggesting the membrane thinning effect of membrane curvature.

\subsection{Cholesterol-Containing Binary Mixtures}

The Cholesterol has been experimentally shown to accumulate in the negatively curved region of the distorted hexagonal phase of the membrane of brominated di(18:0)PC/cholesterol [40]. This prompted us to examine cholesterol-containing binary mixtures. As expected, a DPPC/cholesterol 0.5:0.5 binary mixture, when simulated in a standard planar membrane, formed a pure $\mathrm{L}_{\mathrm{o}}$ domain (data not shown).
When the same mixture was simulated in the $\lambda$ system with a strong curvature, all planar subregions were filled by $\mathrm{L}_{\mathrm{o}}$ domains (set 12, Figure 6(a) and data not shown), whereas the curved and semi-curved subregions contained cholesterol at $53.0 \mathrm{~mol} \%$, which is comparable to $53.8 \mathrm{~mol} \%$ in the planar region (Table 1 and Figure 6(b)). At least this result does not show the accumulation or curvature-sorting of cholesterol in the negatively curved region. While the curved region showed a composition that would form the $\mathrm{L}_{\mathrm{o}}$ domains in a planar membrane, the local properties of the curved region showed small values of the thickness $(\sim 1.7-1.9 \mathrm{~nm})$ and the order parameter $(\sim 0.4-0.5)$, although they were greater than our definition of the $\mathrm{L}_{\mathrm{d}}$ domain (Figure 6(c)). When a DOPC/cholesterol 0.5:0.5 mixture was used in the $\lambda$ system, the sorting was also not significant (Figure 6(e)).

\section{DISCUSSION}

A CG MD simulations were performed on 12 or $20 \mu \mathrm{s}$ scales in a branched bilayer membrane mimicking a hemifusion intermediate. Binary [DOPC/DPPC, DOPE/ DPPC and DPPC/di(18:2)PC] and ternary [DPPC/ di(18:2)PC/cholesterol, DPPC/DOPC/cholesterol] mixtures were examined. Furthermore, binary mixtures of DOPC/cholesterol and DPPC/cholesterol were examined. Owing to limited computational power, the number of simulation runs for each mixture was insufficient for a rigorous statistical assessment, but mutual comparison of the simulations supports the following discussion of the relevance, significance and limitations of this study.

\subsection{Binary Mixtures of Phospholipids}

For the binary DPPC/DOPE mixture (set 1), the negatively curved subregions (at $C=-0.8 \mathrm{~nm}^{-1}$ ) exhibited a DOPE density $\sim 2.2$ times of that in the planar subregion at $20 \mu \mathrm{s}$. The DPPC/DOPE mixture exhibited a higher propensity for curvature-sorting than the DPPC/DOPC mixture, which showed 1.2-fold DOPC enrichment in the curved region compared with the planar region (set 2). Several factors may have contributed to this difference. First, phosphatidylethanolamine (PE), which has hydrogen bond doners and acceptors, has stronger headgroup-headgroup interactions than PC. Second, DOPE has a more negative spontaneous curvature than DOPC. For the CG model used, our estimation using a lipid monolayer/octane/water system at $323 \mathrm{~K}$ showed $C$ $\left( \pm\right.$ s.d) values of $-0.29( \pm 0.05) \mathrm{nm}^{-1}$ for DOPE, -0.19 $( \pm 0.01) \mathrm{nm}^{-1}$ for DOPC, $-0.12( \pm 0.01) \mathrm{nm}^{-1}$ for DPPC, and $-0.28( \pm 0.02) \mathrm{nm}^{-1}$ for $\operatorname{di}(18: 2) \mathrm{PC}$. (We also obtained $C$ values of $-0.22 \pm 0.03 \mathrm{~nm}^{-1}$ for a DOPC/cholesterol 0.7:0.3 mixture and $-0.10 \pm 0.02 \mathrm{~nm}^{-1}$ for a 
DPPC/cholesterol 1:1 mixture ( $\mathrm{L}_{\mathrm{o}}$ domain, 295K). The $C$ value of the CG-cholesterol was estimated as $-0.30 \pm$ $0.01 \mathrm{~nm}^{-1}$ by assuming the linear combination rule to the DOPC/cholesterol data.) Thus, the difference in $C$ between DOPE and DOPC is smaller than the experimental results showing $C=-0.11 \mathrm{~nm}^{-1}$ for DOPC (with tetradecane) and $-0.35 \mathrm{~nm}^{-1}$ for DOPE (Table 1 of [41]) The di(18:2)PC/DPPC membrane (set 3) exhibited a pronounced curvature-sorting of $\operatorname{di}(18: 2) \mathrm{PC}$ (1.9-fold difference between the curved and planar subregions), underscoring the role of spontaneous curvature in lipid sorting. Thus, when the degree of unsaturation differs significantly among constituents [e.g., di(18:2)PC vs DPPC], specific headgroup-headgroup interactions are unnecessary for curvature-induced lipid demixing.

Overall, the results for the binary mixtures can be ex- plained by a theoretical model $[20,42]$ that accounts for the free energy by a tradeoff between two main factors: (1) the entropy cost of demixing and (2) curvature fitness based on spontaneous curvature of individual lipid molecules. Also important is (3) lipid-lipid interaction, which can expedite demixing [43]. The use of strongly interacting lipids such as PE should be equivalent to using lipids with large surface area and is predicted to promote segregation (Eq.1, below). (3) is not limited to specific headgroup-headgroup interactions, but is influenced by overall molecular shape.

When (1) and (2) dominate, the following equation predicts the lipid distribution at the energy minimum [20]. To adapt this equation to our system, we assumed the curved region of our system to be a tube pulled from the liposomes described in [20].
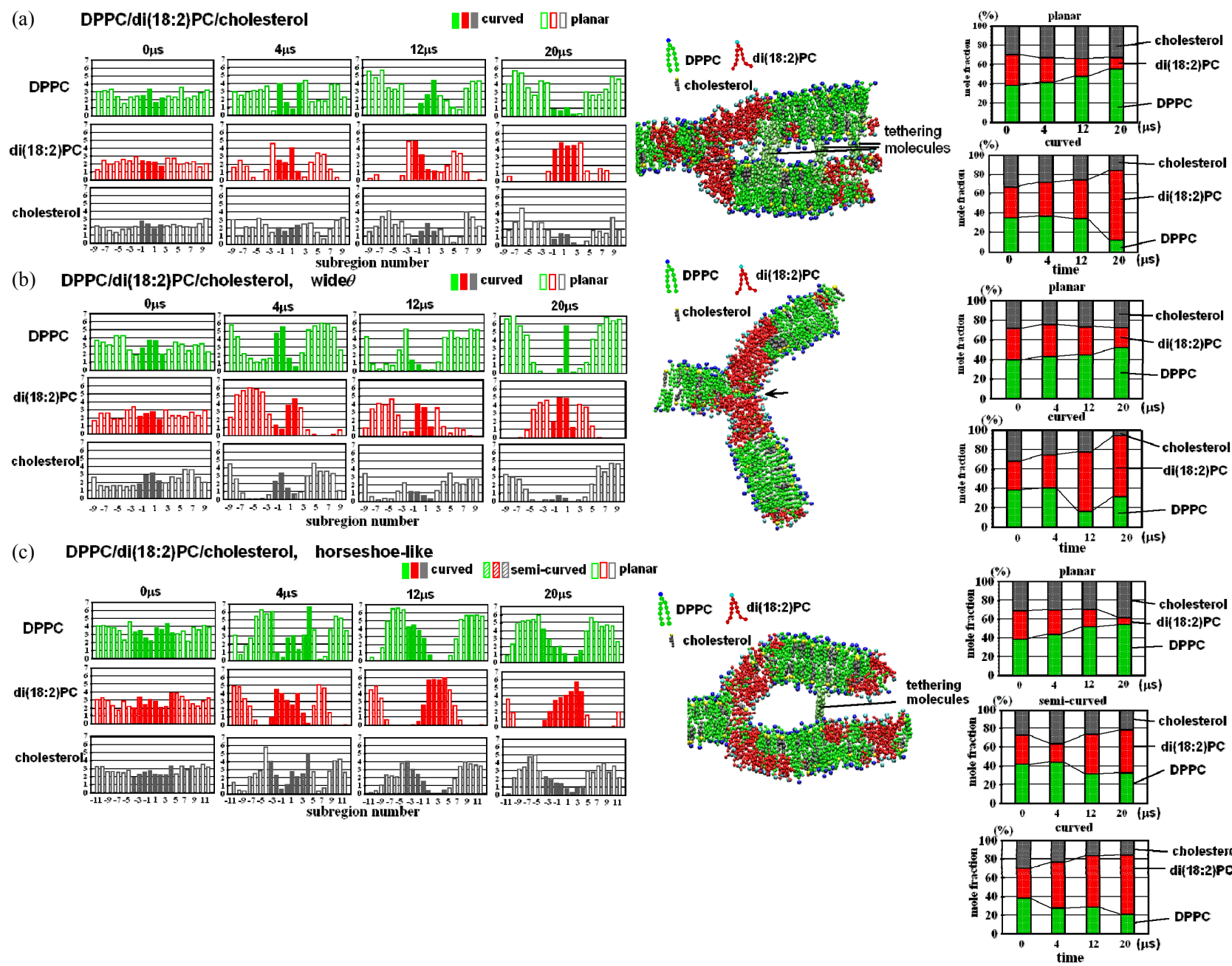

Figure 5. Lipid distribution of the ternary lipid mixtures in the $\lambda$ system. Presentation scheme is the same as in Figure 4, but cholesterol molecules are represented by a yellow sphere (OH group particle) and gray spheres (non-OH particles). (a) DPPC/di(18:2)PC/ cholesterol mixture (set 6); (b) Mixture of the same composition as (a) but tethering molecules were removed and $\theta$ became wide (set 8). Arrow shows DPPC molecules located at the branching point of the membrane; (c) Same mixture as (a), but the $\lambda$ system had a horseshoe-like (weak) curvature (set 10 of Table 1). 

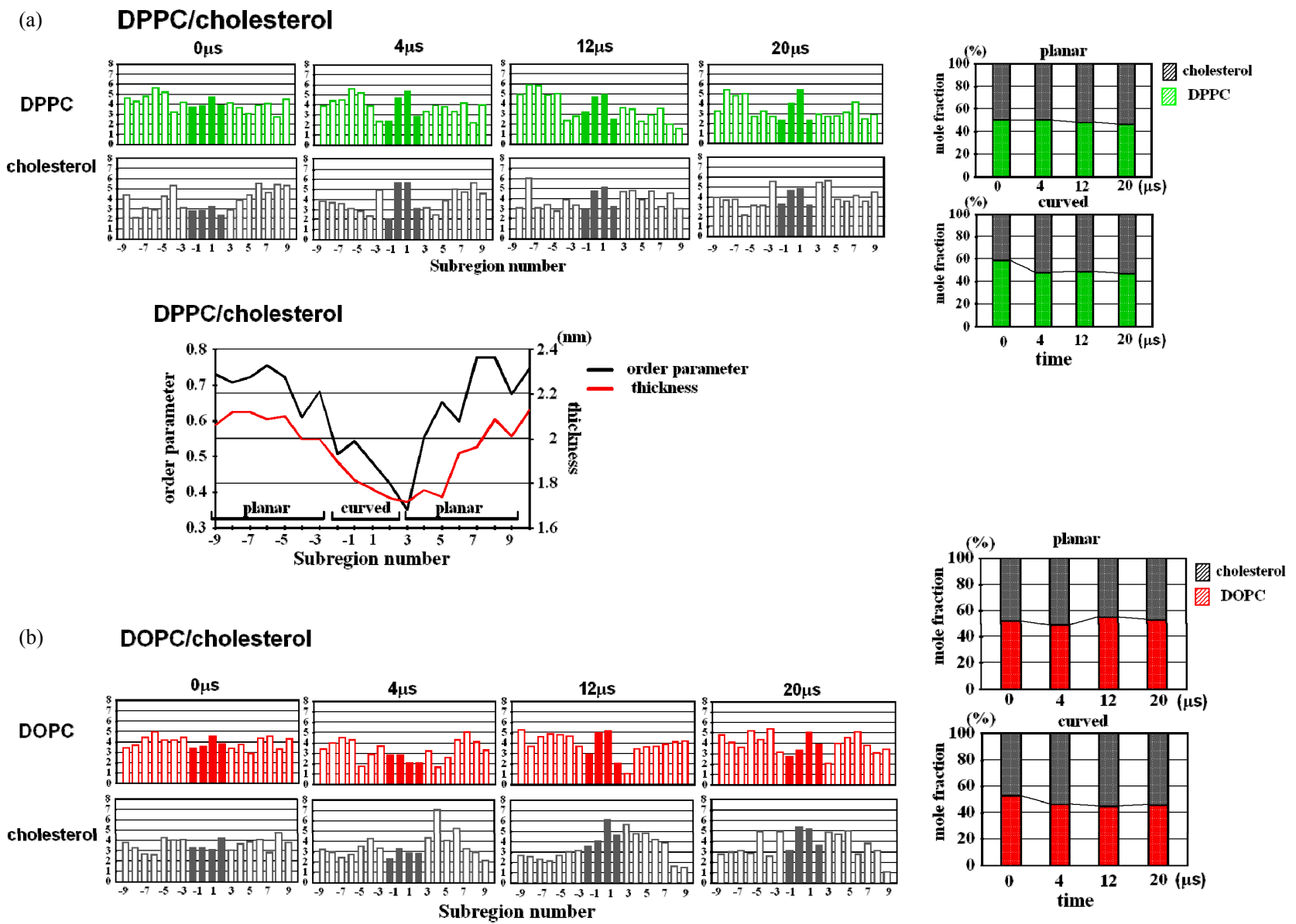

Figure 6. Simulation results of cholesterol-containing binary mixtures. (a) DPPC/cholesterol mixture (set 12). Lipid number of each subregion (top, left), time development of mole fractions (top, right) and thickness and order parameter profiles (bottom) are shown; (b) DOPC/cholesterol mixture (set 13). Lipid number of each subregion (left) and time development of mole fractions (right) are shown.

$$
\begin{aligned}
& k_{c} a\left(C-C_{s t}+C_{s v}\right)\left(C_{\beta}-C_{\alpha}\right) \\
& =k T \ln \left(\phi_{\alpha o v} / \phi_{\alpha o t}\right)+k T \ln \left(\phi_{\beta o t} / \phi_{\beta o v}\right)
\end{aligned}
$$

where, $k_{c}$ is the bending stiffness, $a$ is the molecule area, $C=1 / R_{t}$ is the tube curvature (with $R_{t}$ being the tube radius), $C_{s t}$ and $C_{s v}$ are the spontaneous curvature of the outer monolayers of the tube and the vesicle, respecttively, $k$ is the Boltzmann constant, and $\phi_{\alpha o v}$ is the mole fraction of molecule $\alpha$ in the outer leaflet of the vesicle. Eq.1 was derived via partial differentiation of the total energy function by $N_{\alpha o t}$, which is the number of molecules of $\alpha$ in the outer monolayer of the tube. The left side of Eq.1 corresponds to the potential regarding the bending energy, i.e., (2), whereas the right side corresponds to the entropy term, i.e., (1). The weighted average spontaneous curvature is assumed to be $C_{s t}=\left(C_{\alpha}\right.$ $\left.N_{\alpha o t}+C_{\beta} N_{\beta o t}\right) / N_{o t}$, where $C_{\alpha}$ is the spontaneous curvature of molecule $\alpha$. For our case, Eq.1 is useful as it accounts for the different curvature of the outer and inner leaflets. Minor and major constituents are represented by $\alpha$ and $\beta$ respectively. The $k_{c}$ values for the CG lipids were measured by Fourier analysis of bilayer membranes (see below). If PE-PE interaction is taken into account, Eq.1 explains the observed analyses of the binary phospholipids mixtures well, as we discussed in [19].

\subsection{Ternary Mixtures of Cholesterol/ Saturated Lipid/Unsaturated Lipid (Strong Segregation Limit)}

What factors are important for curvature-sorting of cholesterol-containing ternary mixtures? The model membrane of the DPPC/di(18:2)PC/cholesterol 0.42:0.28:0.3 phase separates into raft-like $\mathrm{L}_{\mathrm{o}}$ and non-raft $\mathrm{L}_{\mathrm{d}}$ domains [34]. In our analysis, the $\mathrm{L}_{\mathrm{o}}$ domains were preferentially located in the planar region, whereas the $\mathrm{L}_{\mathrm{d}}$ domains appeared in the negatively curved region (Figure 5). Several factors can be considered important for demix- 
ing. First, the aforementioned three factors hold here: 1) the entropy cost of demixing; 2) lipid sorting by intrinsic curvature of individual lipids; and 3) lipid-lipid interactions. In addition, 4) the bending stiffness of each domain is likely to be important, (e.g., [14,20]).

Tian and Baumgart [20] also modeled cases in which (1) and (4) are dominant, i.e., curvature sorting on the basis of the bending stiffness are dominant, using the equation,

$$
\begin{aligned}
& 0.5 a k_{c t}^{2} C^{2}\left\{\left(1 / k_{\beta}\right)-\left(1 / k_{\alpha}\right)\right\} \\
& =k T \ln \left(\phi_{\alpha o v} / \phi_{\alpha o t}\right)+k T \ln \left(\phi_{\beta o t} / \phi_{\beta o v}\right),
\end{aligned}
$$

where $k_{c t}$ is the bending stiffness of the tube, which can be modeled as the weighted average of $k_{\alpha}$ and $k_{\beta}$ that are the bending stiffnesses of a minor (and stiff) constituent $\alpha$ and the major constituent $\beta$, respectively, and the other variables same as in Eq.1. Let us suppose that $\alpha$ is a minor constituent forming a small raft-like $\mathrm{L}_{\mathrm{o}}$ domain (of $<1 \mathrm{~nm}$ ), and the remaining constituents $\beta$ represent lipids that form non-raft $\mathrm{L}_{\mathrm{d}}$ domains. Our Fourier analysis based on the method similar to $[44,45]$ showed that the bending stiffness $k_{c}$ of a DPPC/cholesterol bilayer is $2.2( \pm 0.3) \times 10^{-20} \mathrm{~J}$, whereas that of a DOPC bilayer is $1.5( \pm 0.2) \times 10^{-20} \mathrm{~J}$ and that of a di(18:2)PC bilayer is $7.5 \pm 0.5 \times 10^{-21} \mathrm{~J}$. These are smaller than those reported by [37] likely because of differences in the procedures for the binning and smoothing for the Fourier analysis, but in any case, $k_{c}$ of the $\mathrm{L}_{\mathrm{o}}$ domain of the CG DPPC/ cholesterol was only three times as large as that of the di(18:2)PC bilayer. Obviously, near-depletion of the minor component $\alpha$ (here, DPPC/cholesterol) from the curved region would bring $\phi_{\alpha o t}$ close to zero, inflating the right side of Eq.2. Furthermore, even if $k_{\alpha}$ increases, the left side of Eq.2 grows only modestly [20].

These considerations suggest that, during an early phase of demixing (i.e., when the domains are small), the rigidity difference between the $L_{o}$ and $L_{d}$ domains would be roughly as effective for curvature-sorting as the spontaneous curvature difference (as considered above). However, once the domains have become large (e.g. $>\sim 1 \mathrm{~nm}$ ), extensive demixing associated with the domain coalescence would occur (e.g., Figure 4(a) and (b)). This can be considered as a lipid-lipid interaction (3) that increases the effective area per molecule (i.e., $a$ in both Eq.1 and Eq.2). In fact, by simply applying DPPC (plus cholesterol) and DPPC fractions of set 8 to $\beta$ and $\alpha$, respectively, of Eq.2 and by using the rigidity reported by [37], one obtains too large a value of $a=\sim 1 \mathrm{~nm}^{2}$, suggesting that heterogeneity in rigidity cannot explain the data without the assist of lipid-lipid interaction. Of note, Eq.1 alone cannot explain the set 8 result, either; by applying set 8 data for Eq.1, $a$ becomes $\sim 2 \mathrm{~nm}^{2}$, which is too large, given the small area per molecule of cholesterol.

Another factor that should be considered is (5) the force leading to interleaflet colocalization (i.e, registration), which makes the $\mathrm{L}_{\mathrm{o}}$ domains symmetric between two contacting leaflets [34]. A pitfall of the $\lambda$ system in this respect is that it consists of both bilayer and non-bilayer parts; the $\mathrm{L}_{\mathrm{o}}$ domains that formed symmetrically in planar regions should stabilize each other, helping to bring the $\mathrm{L}_{\mathrm{o}}$ domains into the planar regions (Figures 5 and 7 and unpublished results). This issue must be addressed by many more simulations with different settings. Another potentially important factor is that (6) locally asymmetric domain formation may generate abrupt bending of bilayer membranes. Even if the lipid composition is symmetric between the two leaflets of a bilayer, incomplete inter-leaflet registration of domains may lead to an abrupt bending. Intriguingly, in some simulations, the boundary between a raft-like and non-raft domain moved to and stayed at the branched point of the $\lambda$ membrane (Figure 7). Biological membranes are likely to be poised between the mixing and demixing phases, providing a basis on which even slight changes in collective behavior may have profound effects on membrane curvature $[10,12,14]$.

One might ask whether the $\lambda$ system can be used to analyze the weak segregation limit. It has been shown that when the lipid composition is near the mixing/ demixing (miscibility) critical points [46], the curvature amplifies the lipid demixing, as demonstrated in thin tube formation from liposomes [12,14,20]. However, our experience suggests that statistical uncertainties make rigorous analysis of such weak segregation limits using standard CG simulations difficult (unpublished results).

\subsection{Binary Cholesterol/Phospholipid Mixture}

Cholesterol not only forms rigid microdomains that are necessary for organizing fusion proteins into the fusion site, but also facilitates various steps in the fusion process, likely by staying free from such microdomains $[8,23,47,48]$. Fratti et al. have shown that the periphery of the hemifusion diaphragm (the vertices) is enriched in ergosterol and DAG, both of which have intrinsic negative curvature [48]. However, our results of the DPPC/cholesterol and DOPC/cholesterol binary mixtures did not show significant cholesterol enrichment in the curved region in compared with the planar region (set 12 and 13, Figure 6). Considering the limitations in the correctness of CG modeling, all-atom simulations may help determine to what extent cholesterol is enriched in the curved regions under the conditions close to experiments. 
DPPC/di(18:2)PC/cholesterol, set 8
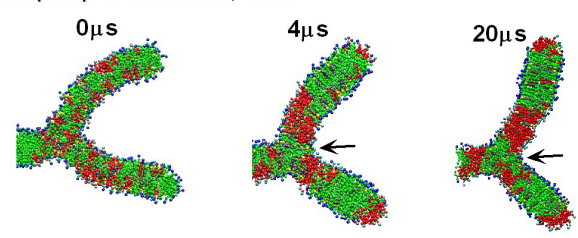

(a)
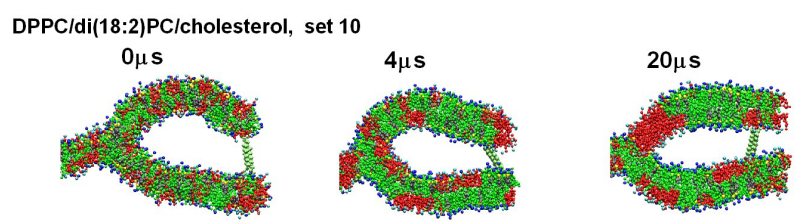

(b)

Figure 7. Examples of the cases in which a domain boundary formed at the membrane branching point. Side view is shown using the scheme as in Figure 4. (a) and (b) are snapshots from set 8 and 10, respectively. Arrow in (a) shows DPPC molecules located at the branching point of the membrane.

\subsection{Technical Limitations and Other Notable Findings}

The limitations of our system should be considered. First, the $\lambda$ membrane has a two-dimensional curvature, lacking Gaussian curvature. Care must be taken if our findings are extrapolated to hemifusion intermediates, particularly small intermediates that are likely to originnate at a single point of the apposed membrane surfaces. Note that a vertex ring hemifusion model has been proposed by [49]. Second, the use of tethering molecules is an artificial perturbation. Another limitation concerns the dimension of the membrane. We adopted a membrane that is short in the $y$-direction, but this leads to rapid formation of a stripe pattern, which is an artifact of the finite size effect. In two additional simulations we doubled the thickness in the $y$-direction, yet the stripe pattern still formed.

A notable feature of our results is the horseshoe-like structure that occurs upon removal of the proximal tethering molecules (set 10). This curvature $\left(C=\sim-0.6 \mathrm{~nm}^{-1}\right)$ appears weaker than that derived by the self-consistent field analysis by Schick and coworkers [50,51]. We reason that this discrepancy arises in part from the difference in the bending stiffness $k_{c}$, which may be generally greater for the atomistic and $\mathrm{CG}$ models than for the copolymer models in [50]. Another reason could be the difference in the energy required for separation of the two cis monolayers. The two cis monolayers of the planar subregions separate in our study, implying a weak attractive force between them, whereas the monolayers appear to interact quite strongly even near the branching point in [50]. Direct measurement of the tension imposed on the tethering molecules could be informative.
We also note that, lipid partition at the branching point is influenced not only by curvature itself but also by a favorable membrane thickness. The curved subregions often contained DPPC as minor components in addition to DOPE, DOPC, or di(18:2)PC (arrows in Figure 5(b) and Figure 7(a)). Long phospholipids near the void created by three monolayers could be favored because they can fill the void better.

\subsection{Hemifusion and Perspectives of Lipid Distribution Analysis}

Recent studies on exocytosis have suggested or provided evidence for hemifusion intermediates in living cells. A flickering fusion pore, which supports the presence of a hemifusion intermediate, has been studied extensively (e.g., [52]). Kiss-and-run exocytosis, which is likely to be mediated by hemifused states, has been studied in many systems (e.g., [53-57]). Furthermore, visualization of hemifusion intermediates by conical electron tomography provided direct evidence of hemifusion [58]. Vacuoles isolated from yeast allowed observations of hemifusion and full fusion in a kinetically separated manner [49]. Also, in several liposome fusion experiments, hemifusion has been shown to be an on-pathway intermediate. For POPC/DOPS liposomes containing yeast $\mathrm{v}$ - and t-SNAREs [59], a fluorescence resonance energy transfer (FRET) plateau suggestive of hemifusion was observed, and in addition, a gradual FRET increase often preceded hemifusion, suggesting the involvement of partially restricted hemifusion. With further technical improvement, hemifusion might be implicated even in cases previously considered to indicate a direct transition from liposome adhesion to full fusion (e.g., [60,61]). Considering the time resolution $(>\mathrm{ms})$ of such studies, a rearrangement of the lipid distribution is likely to occur in hemifused membranes.

The effects of lipid composition on the kinetics and outcomes of fusion have also been studied. PE modulates the fusion kinetics and outcomes in fusion experiments and simulations (e.g., [62,63]). In liposome experiments using PC, PS, PE, t-SNAREs and v-SNAREs [64], an increase in PE stabilizes the hemifusion intermediates, slowing the transition to full fusion. Several experimental, computational and theoretical analyses also highlighted the importance of the PC:PE ratio to free energy of hemifusion intermediates, leading to a general consensus that a high density of PE (or other negative-curvature lipids) in the proximal leaflets promotes stalk formation and stabilizes hemifusion intermediates $[28,50,65]$. The PE-stabilized hemifusion intermediates may provide an environment suitable for regulated exocytosis via flickering or kiss-and-run events. 
Our approach may be particularly useful in analyzing fusion of largely flat membranes; fusion simulations mainly use highly curved membranes (vesicles with a 15 $20 \mathrm{~nm}$ diameter), whereas large (small curvature) vesicles tend to lead to hemifusion and static adhesion [66]. Although we have not examined them, lipids having strong negative curvature, such as phosphatidic acid and DAG could stabilize hemifusion intermediate at low concentrations, easing the intricate regulation of duration of the hemifused states.

Finally, this study focuses on model membranes, but the mechanisms for phase separation in cellular membranes may be different from that in the model membranes. The $\mathrm{L}_{\mathrm{o}}$ and $\mathrm{L}_{\mathrm{d}}$ phases formed in model membranes usually exhibit more pronounced differences in the degree of lipid order than phases formed in vesicles prepared from plasma membranes [35]. This small difference in the degree of packing in plasma membranes suggests important roles for protein-protein and protein-lipid interactions, headgroup-headgroup interactions between SM molecules, and carbohydrate chain interacttions in phase separations of plasma membranes [35]. Clearly, the biological relevance of our results must be evaluated carefully. Many more experimental and computational efforts are necessary in order to gain insights into the roles of lipid redistribution in membrane fusion and intracellular lipid sorting.

\section{ACKNOWLEDGEMENTS}

We thank the anonymous reviewers for helpful comments. This work was supported by a Grant-in-Aid for Scientific Research from the Ministry of Education, Culture, Sports, Science, and Technology of Japan.

\section{REFERENCES}

[1] Van Meer, G., Voelker, D.R. and Feigenson, G.W. (2008) Membrane lipids: Where they are and how they behave. Nature Reviews Molecular Cell Biology, 9, 112-124. doi: $10.1038 / \mathrm{nrm} 2330$

[2] Simons, K. and Ikonen, E. (1997) Functional rafts in cell membranes. Nature, 387, 569-572. doi:10.1038/42408

[3] Jacobson, K., Mouritsen, O.G. and Anderson, R.G. (2007) Lipid rafts: At a crossroad between cell biology and physics. Nature Cell Biology, 9, 7-14. doi:10.1038/ncb0107-7

[4] Chernomordik, L.V. and Kozlov, M.M. (2008) Mechanics of membrane fusion. Nature Structural \& Molecular Biology, 15, 675-683. doi:10.1038/nsmb.1455

[5] Vogel, S.S., Leikina, E.A. and Chernomordik, L.V. (1993) Lysophosphatidylcholine reversibly arrests exocytosis and viral fusion at a stage between triggering and membrane merger. Journal of Biological Chemistry, 268, 25764-25768.

[6] Cohen, F.S. and Melikyan, G.B. (2004) The energetics of membrane fusion from binding, through hemifusion, pore formation, and pore enlargement. Journal of Membrane Biology, 199, 1-14.

doi:10.1007/s00232-004-0669-8

[7] Chernomordik, L.V. and Kozlov, M.M. (2003) Proteinlipid interplay in fusion and fission of biological membranes. Annual Review of Biochemistry, 72, 175-207. doi:10.1146/annurev.biochem.72.121801.161504

[8] Churchward, M.A., Rogasevskaia, T., Höfgen, J., Bau, J. and Coorssen, J.R. (2005) Cholesterol facilitates the native mechanism of $\mathrm{Ca}^{2+}$-triggered membrane fusion. Journal of Cell Science, 118, 4833-4848. doi: $10.1242 /$ jcs.02601

[9] Lingwood, D., Kaiser, H.J., Levental, I. and Simons, K. (2009) Lipid rafts as functional heterogeneity in cell membranes. Biochemical Society Transactions, 37, 955-960. doi:10.1042/BST0370955

[10] Lingwood, D., Ries, J., Schwille, P. and Simons, K. (2008) Plasma membranes are poised for activation of raft phase coalescence at physiological temperature. Proceedings of the National Academy of Sciences of the United States of America, 105, 10005-10010. doi:10.1073/pnas.0804374105

[11] Churchward, M.A. and Coorssen, J.R. (2009) Cholesterol, regulated exocytosis and the physiological fusion machine. Biochemical Journal, 423, 1-14. doi:10.1042/BJ20090969

[12] Roux, A., Cuvelier, D., Nassoy, P., Prost, J., Bassereau, P. and Goud, B. (2005) Role of curvature and phase transition in lipid sorting and fission of membrane tubules. EMBO Journal, 24, 1537-1545. doi:10.1038/sj.emboj.7600631

[13] Baumgart, T., Hess, S.T. and Webb, W.W. (2003) Imaging coexisting fluid domains in biomembrane models coupling curvature and line tension. Nature, 425, 821-824. doi: 10.1038/nature02013

[14] Sorre, B., Callan-Jones, A., Manneville, J.B., Nassoy, P., Joanny, J.F., Prost, J., Goud, B. and Bassereau, P. (2009) Curvature-driven lipid sorting needs proximity to a demixing point and is aided by proteins. Proceedings of the National Academy of Sciences of the United States of America, 106, 5622-5626. doi:10.1073/pnas.0811243106

[15] Parthasarathy, R., Yu, C.H. and Groves, J.T. (2006) Curvature-modulated phase separation in lipid bilayer membranes. Langmuir, 22, 5095-5099. doi:10.1021/la060390o

[16] Mukherjee, S., Soe, T.T. and Maxfield, F.R. (1999) Endocytic sorting of lipid analogues differing solely in the chemistry of their hydrophobic tails. Journal of Cell Biology, 144, 1271-1284. doi:10.1083/jcb.144.6.1271

[17] Van Meer, G. and Lisman, Q. (2002) Sphingolipid transport: Rafts and translocators. Journal of Biological Chemistry, 277, 25855-25858. doi:10.1074/jbc.R200010200

[18] Ostrowski, S.G., Van Bell, C.T., Winograd, N. and Ewing, A.G. (2004) Mass spectrometric imaging of highly curved membranes during Tetrahymena mating. Science, 305, 71-73. doi:10.1126/science.1099791

[19] Nishizawa, M. and Nishizawa, K. (2010) Curvaturedriven lipid sorting: Coarse-grained dynamics simulations of a membrane mimicking a hemifusion intermediate. Journal of Biophysical Chemistry, 1, 86-95. doi:10.4236/jbpc.2010.12011 
[20] Tian, A. and Baumgart, T. (2009) Sorting of lipids and proteins in membrane curvature gradients. Biophysical Journal, 96, 2676-2688. doi:10.1016/j.bpj.2008.11.067

[21] Derganc, J. (2007) Curvature-driven lateral segregation of membrane constituents in Golgi cisternae. Physical Biology, 4, 317-324. doi:10.1088/1478-3975/4/4/008

[22] Cooke, I.R. and Deserno, M. (2006) Coupling between lipid shape and membrane curvature. Biophysical Journal, 91, 487-495. doi:10.1529/biophysj.105.078683

[23] Epand, R.M., Thomas, A., Brasseur, R. and Epand, R.F. (2010) Cholesterol interaction with proteins that partition into membrane domains: An overview. Subcellular Biochemistry, 51, 253-278.

doi:10.1007/978-90-481-8622-8_9

[24] Nielsen, S.O., Lopez, C.F., Srinivas, G. and Klein, M.L. (2004) Coarse grain models and the computer simulations of soft materials. Journal of Physics Condensed Matter, 16, R481-R512. doi:10.1088/0953-8984/16/15/R03

[25] Marrink, S.-J., De Vries, A.H. and Tieleman, D.P. (2009) Lipids on the move: Simulation of membrane pores, domains, stalks and curves. Biochimica et Biophysica Acta, 1788, 149-168. doi:10.1016/j.bbamem.2008.10.006

[26] Marrink, S.J. and Mark, A.E. (2003) The mechanism of vesicle fusion as revealed by molecular dynamics simulations. Journal of American Chemical Society, 125, 11144-11145. doi:10.1021/ja036138+

[27] Kasson, P.M., Kelley, N.W., Singhal, N., Vrljic, M., Brunger, A.T. and Pande, V.S. (2006) Ensemble molecular dynamics yields submillisecond kinetics and intermediates of membrane fusion. Proceedings of the National Academy of Sciences of the United States of America, 103, 11916-11921. doi:10.1073/pnas.0601597103

[28] Kasson, P.M. and Pande, V.S. (2007) Control of membrane fusion mechanism by lipid composition: Predictions from ensemble molecular dynamics. PLoS Computational Biology, 3, e220.

doi:10.1371/journal.pcbi.0030220

[29] Chernomordik, L.V. and Kozlov, M.M. (2005) Membrane hemifusion: Crossing a chasm in two leaps. Cell, 123, 375-382. doi:10.1016/j.cell.2005.10.015

[30] Liu, T., Wang, T., Chapman, E.R. and Weisshaar, J.C. (2008) Productive hemifusion intermediates in fast vesicle fusion driven by neuronal SNAREs. Biophysical Journal, 94, 1303-1314. doi:10.1529/biophysj.107.107896

[31] Niemelä, P.S., Ollila, S., Hyvönen, M.T., Karttunen, M. and Vattulainen, I. (2007) Assessing the nature of lipid raft membranes. PLoS Computational Biology, 3, e34.

[32] Bennett, W.F., MacCallum, J.L., Hinner, M.J., Marrink, S.J. and Tieleman, D.P. (2009) Molecular view of cholesterol flip-flop and chemical potential in different membrane environments. Journal of American Chemical Society, 131, 12714-12720. doi:10.1021/ja903529f

[33] Berkowitz, M.L. (2009) Detailed molecular dynamics simulations of model biological membranes containing cholesterol. Biochimica et Biophysica Acta, 1788, 86-96.

[34] Risselada, H.J. and Marrink, S.J. (2008) The molecular face of lipid rafts in model membranes. Proceedings of the National Academy of Sciences of the United States of America, 105, 17367-17372. doi:10.1073/pnas.0807527105

[35] Kaiser, H.J., Lingwood, D., Levental, I., Sampaio, J.L., Kalvodova, L., Rajendran, L. and Simons K. (2009) Order of lipid phases in model and plasma membranes. Proceedings of the National Academy of Sciences of the United States of America, 106, 16645-16650. doi:10.1073/pnas.0908987106

[36] Lindahl, E., Hess B. and Van der Spoel D. (2001) GROMACS 3.0: A package for molecular simulation and trajectory analysis. Journal of Molecular Modeling, 7, 306-317.

[37] Marrink, S.J., Risselada, H.J., Yefimov, S., Tieleman, D.P. and De Vries, A.H. (2007) The MARTINI force field; coarse grained model for biomolecular simulation. Journal of Physical Chemistry, 111, 7812-7824. doi:10.1021/jp071097f

[38] Berendsen, H.J.C., Postma, J.P.M., Van Gunsteren, W.F., DiNola, A. and Haak, J.R. (1984) Molecular dynamics with coupling to an external bath. Journal of Chemical Physics, 81, 3684-3690. doi:10.1063/1.448118

[39] Humphery W., Dalke A. and Schulten K. (1996) VMDVisual molecular dynamics. Journal of Molecular Graphics and Modelling, 14, 33-38. doi:10.1016/0263-7855(96)00018-5

[40] Wang, W., Yang, L. and Huang, H.W. (2007) Evidence of cholesterol accumulated in high curvature regions: Implication to the curvature elastic energy for lipid mixtures. Biophysical Journal, 92, 2819-2830. doi:10.1529/biophysj.106.097923

[41] Chen, Z. and Rand, R.P. (1997) The influence of cholesterol on phospholipid membrane curvature and bending elasticity. Biophysical Journal, 73, 267-276. doi:10.1016/S0006-3495(97)78067-6

[42] Kozlov, M. M. and Helfrich, W. (1992) Effects of a cosurfactant on the stretching and bending elasticities of a surfactant monolayer. Langmuir, 8, 2792-2797. doi:10.1021/1a00047a035

[43] Kamal, M.M., Mills, D., Grzybek, M. and Howard, J. (2009) Measurement of the membrane curvature preference of phospholipids reveals only weak coupling between lipid shape and leaflet curvature. Proceedings of the National Academy of Sciences of the United States of America, 106, 22245-22250. doi:10.1073/pnas.0907354106

[44] Marrink, S.J. and Mark, A.E. (2001) Effect of undulations on surface tension in simulated bilayers. Journal of Physical Chemistry B, 105, 6122-6127.

[45] Lindahl, E. and Edholm, O. (2000) Mesoscopic undulations and thickness fluctuations in lipid bilayers from molecular dynamics simulations. Biophysical Journal, 79, 426-433.

[46] Veatch, S.L. and Keller, S.L. (2003) Separation of liquid phases in giant vesicles of ternary mixtures of phospholipids and cholesterol. Biophysical Journal, 85, 3074-3083. doi:10.1016/S0006-3495(03)74726-2

[47] García, R.A., Pantazatos, S.P., Pantazatos, D.P. and MacDonald, R.C. (2001) Cholesterol stabilizes hemifused phospholipid bilayer vesicles. Biochimica et Biophysica Acta, 1511, 264-270.

[48] Fratti, R.A., Jun, Y., Merz, A.J., Margolis, N. and Wickner, W. (2004) Interdependent assembly of specific regulatory lipids and membrane fusion proteins into the ver- 
tex ring domain of docked vacuoles. Journal of Cell Biology, 167, 1087-1098. doi:10.1083/jcb.200409068

[49] Jun, Y. and Wickner, W. (2007) Assays of vacuole fusion resolve the stages of docking, lipid mixing, and content mixing. Proceedings of the National Academy of Sciences of the United States of America, 104, 13010-13015. doi:10.1073/pnas.0700970104

[50] Katsov, K., Müller, M. and Schick, M. (2004) Field theoretic study of bilayer membrane fusion. I. Hemifusion mechanism. Biophysical Journal, 87, 3277-3290. doi:10.1529/biophysj.103.038943

[51] Lee, J.Y. and Schick, M. (2007) Field theoretic study of bilayer membrane fusion III: Membranes with leaves of different composition. Biophysical Journal, 92, 3938-3948. doi:10.1529/biophysj.106.097063

[52] Staal, R.G., Mosharov, E.V. and Sulzer, D. (2004) Dopamine neurons release transmitter via a flickering fusion pore. Nature Neuroscience, 7, 341-346. doi:10.1038/nn1205

[53] Aravanis, A.M., Pyle, J.L. and Tsien, R.W. (2003) Single synaptic vesicles fusing transiently and successively without loss of identity. Nature, 423, 643-647. doi:10.1038/nature 01686

[54] Vardjan, N., Stenovec, M., Jorgacevski, J., Kreft, M. and Zorec, R. (2007) Subnanometer fusion pores in spontaneous exocytosis of peptidergic vesicles. Journal of Neuroscience, 27, 4737-4746. doi:10.1523/JNEUROSCI.0351-07.2007

[55] Xia, X., Lessmann, V. and Martin, T.F. (2009) Imaging of evoked dense-core-vesicle exocytosis in hippocampal neurons reveals long latencies and kiss-and-run fusion events. Journal of Cell Science, 122, 75-82. doi: $10.1242 /$ jes. 034603

[56] Hanna, S.T., Pigeau, G.M., Galvanovskis, J., Clark, A., Rorsman, P. and MacDonald, P.E. (2009) Kiss-and-run exocytosis and fusion pores of secretory vesicles in human beta-cells. Pflugers Arch, 457, 1343-1350. doi:10.1007/s00424-008-0588-0

[57] Miklavc, P., Albrecht, S., Wittekindt, O.H., Schullian, P., Haller, T. and Dietl, P. (2009) Existence of exocytotic hemifusion intermediates with a lifetime of up to seconds in type II pneumocytes. Biochemical Journal, 424, 7-14. doi:10.1042/BJ20091094
[58] Zampighi, G.A., Zampighi, L.M., Fain, N., Lanzavecchia, S., Simon, S.A. and Wright, E.M. (2006) Conical electron tomography of a chemical synapse: Vesicles docked to the active zone are hemi-fused. Biophysical Journal, 91, 2910-2918. doi:10.1529/biophysj.106.084814

[59] Yoon, T.Y., Okumus, B., Zhang, F., Shin, Y.K. and Ha, T. (2006) Multiple intermediates in SNARE-induced membrane fusion. Proceedings of the National Academy of Sciences of the United States of America, 103, 1973119736. doi:10.1073/pnas.0606032103

[60] Floyd, D.L., Harrison, S.C. and van Oijen, A.M. (2009) Method for measurement of viral fusion kinetics at the single particle level. Journal of Visualized Experiments, 1484.

[61] Wang, T., Smith, E.A., Chapman, E.R. and Weisshaar, J.C. (2009) Lipid mixing and content release in single-vesicle, SNARE-driven fusion assay with 1 - $5 \mathrm{~ms}$ resolution. Biophysical Journal, 96, 4122-4131. doi:10.1016/j.bpj.2009.02.050

[62] Haque, M.E., McIntosh, T.J. and Lentz, B.R. (2001) Influence of lipid composition on physical properties and peg-mediated fusion of curved and uncurved model membrane vesicles: "nature's own" fusogenic lipid bilayer. Biochemistry, 40, 4340-4348. doi:10.1021/bi002030k

[63] Emoto, K. and Umeda, M. (2000) An essential role for a membrane lipid in cytokinesis. Regulation of contractile ring disassembly by redistribution of phosphatidylethanolamine. Journal of Cell Biology, 149, 1215-1224. doi: $10.1083 /$ jcb.149.6.1215

[64] Liu, T, Tucker, W.C., Bhalla, A., Chapman, E.R. and Weisshaar, J.C. (2005) SNARE-driven, 25-millisecond vesicle fusion in vitro. Biophysical Journal, 89, 24582472. doi:10.1529/biophysj.105.062539

[65] Siegel, D.P. and Epand, R.M. (1997) The mechanism of lamellar-to-inverted hexagonal phase transitions in phosphatidylethanolamine: Implications for membrane fusion mechanisms. Biophysical Journal, 73, 3089-3111. doi:10.1016/S0006-3495(97)78336-X

[66] Grafmüller, A., Shillcock, J. and Lipowsky, R. (2007) Pathway of membrane fusion with two tension-dependent energy barriers. Physical Review Letter, 98, 21810. 